\title{
Generation and screening of a comprehensive Mycobacterium avium subsp. paratuberculosis transposon mutant bank
}

\author{
Govardhan Rathnaiah ${ }^{1}$, Elise A. Lamont ${ }^{2}$, N. Beth Harris ${ }^{1}$, Robert J. Fenton ${ }^{1}$, Denise K. Zinniel ${ }^{1}$, \\ Xiaofei Liu ${ }^{1}$, Josh Sotos ${ }^{3}$, Zhengyu Feng ${ }^{1}$, Ayala Livneh-Kol ${ }^{4}$, Nahum Y. Shpigel ${ }^{4}$, \\ Charles J. Czuprynski ${ }^{3}$, Srinand Sreevatsan ${ }^{2}$ and Raúl G. Barletta ${ }^{1 *}$
}

'School of Veterinary Medicine and Biomedical Sciences, University of Nebraska, Lincoln, NE, USA

${ }^{2}$ Department of Veterinary Population Medicine, University of Minnesota, St. Paul, MN, USA

${ }^{3}$ School of Veterinary Medicine, University of Wisconsin, Madison, WI, USA

${ }^{4}$ The Koret School of Veterinary Medicine, The Hebrew University of Jerusalem, Rehovot, Israel

\section{Edited by:}

Thomas A. Ficht, Texas A\&M

University, USA

Reviewed by:

Thomas C. Zahrt, Medical College of Wisconsin, USA

Jeffrey Cirillo, Texas A\&M Health

Science Center, USA

*Correspondence:

Raúl G. Barletta, School of Veterinary

Medicine and Biomedical Sciences,

University of Nebraska, 211 VBS,

Fair Street and East Campus Loop,

Lincoln, NE 68583-0905, USA

e-mail: rbarletta@unl.edu
Mycobacterium avium subsp. paratuberculosis (MAP) is the etiologic agent of Johne's Disease in ruminants. This enteritis has significant economic impact and worldwide distribution. Vaccination is one of the most cost effective infectious disease control measures. Unfortunately, current vaccines reduce clinical disease and shedding, but are of limited efficacy and do not provide long-term protective immunity. Several strategies have been followed to mine the MAP genome for virulence determinants that could be applied to vaccine and diagnostic assay development. In this study, a comprehensive mutant bank of 13,536 MAP K-10 Tn5367 mutants $(P>95 \%)$ was constructed and screened in vitro for phenotypes related to virulence. This strategy was designated to maximize identification of genes important to MAP pathogenesis without relying on studies of other mycobacterial species that may not translate into similar effects in MAP. This bank was screened for mutants with colony morphology alterations, susceptibility to D-cycloserine, impairment in siderophore production or secretion, reduced cell association, and decreased biofilm and clump formation. Mutants with interesting phenotypes were analyzed by PCR, Southern blotting and DNA sequencing to determine transposon insertion sites. These insertion sites mapped upstream from the MAP1152-MAP1156 cluster, internal to either the Mod operon gene MAP1566 or within the coding sequence of Isr2, and several intergenic regions. Growth curves in broth cultures, invasion assays and kinetics of survival and replication in primary bovine macrophages were also determined. The ability of vectors carrying Tn5370 to generate stable MAP mutants was also investigated.

Keywords: Johne's Disease, Mycobacterium paratuberculosis, transposon, mutant bank, bovine macrophages

\section{INTRODUCTION}

Mycobacterium avium subsp. paratuberculosis (MAP) is the etiologic agent of Johne's Disease (JD) in ruminants. This enteritis has significant economic impact and worldwide distribution (Sweeney, 1996). In the United States, annual losses to the dairy industry have been estimated from 250 million (Ott et al., 1999) to $\$ 1.5$ billion (Stabel, 1998). Vaccination is one of the most cost effective disease control measures. Unfortunately, though there are JD vaccines that reduce clinical disease and shedding, their efficacies are limited and none afford long-term protective immunity. For example in the United States, Mycopar ${ }^{\circledR}$ (Boehringer

Abbreviations: CAS, Chrome azurol S; CFU, Colony forming units; DCS, Dcycloserine; Hyg, Hygromycin; JD, Johne's Disease; Kan, Kanamycin; MAP, Mycobacterium avium subsp. paratuberculosis; MDM, Monocyte-derived macrophage; MOADC, Middlebrook 7H9 broth enriched with oleic acid albumin dextrose complex; Msmeg, Mycobacterium smegmatis; MTB, Mycobacterium tuberculosis; OD, Optical density; ORF, Open reading frame; PBS, Phosphate buffered saline; SEM, Standard error of the mean.
Ingelheim Vetmedica, Inc.) is the only licensed vaccine against JD. However, this vaccine is derived from M. avium Strain 18 (Bastida and Juste, 2011), and therefore does not have an optimal antigenic repertoire. Another bacterin, Silirum ${ }^{\circledR}$ (Zoetis Animal Health) is being tested in Australia and approved for limited use. It is a heat-killed MAP vaccine strain with improved safety. This formulation may possess a better antigenic repertoire but heat-killing may reduce efficacy. Neoparasec ${ }^{\circledR}$ (Rhone-Merieux) contains the live-attenuated MAP strain $316 \mathrm{~F}$ while Gudair ${ }^{\circledR}$ (Zoetis Animal Health) is heat-killed $316 \mathrm{~F}$ and licensed for use in sheep and goats. However, current vaccines cannot distinguish vaccinated from infected animals, thus compromising JD diagnostic tests (Hines et al., 2014), and strain 316F was generated in the 1920's by random attenuation procedures (e.g., passages on ox bile) and their attenuating mutations are only now being investigated (Bull et al., 2013). In last analysis, a vaccine of high efficacy is needed for an effective control of JD (Lu et al., 2013). 
The MAP wild type strain K-10 genome has been sequenced, annotated and reannotated by optical mapping (Li et al., 2005; Wu et al., 2009). The updated K-10 genome is represented by a circular map of $4,829,781$ bp encoding 4350 open reading frames (ORFs) with 69.3\% GC content. In this genome, about 60\% of the ORFs have known homologs in databases but only $30 \%$ have definitive function predictions (Bannantine et al., 2012). Several strategies have been followed to mine the MAP genome for virulence determinants or antigens of diagnostic importance (Bannantine and Paustian, 2006; Cho et al., 2007; Li et al., 2007). One approach relied on identifying MAP genes with known M. tuberculosis (MTB) homologs or orthologs (Sampson et al., 2004; Bach et al., 2006). For example, based on the attenuation of MTB leuD mutants (Sampson et al., 2004), the corresponding MAP leuD mutants were constructed by allelic exchange, characterized by carbon and nitrogen source utilization, showed to be attenuated in mice (Chen et al., 2012a,b), and provide protection against wild type challenge in goats (Faisal et al., 2013). Indeed, differences in gene organization may lead to contextdependent function: e.g., even homologous genes in MAP and M. avium subsp. hominissuis (MAH) may play different roles in pathogenesis (Wu et al., 2006). In addition, genomic differences may be associated with specific hosts (Bannantine et al., 2012). Thus, strategies based on gene homology are not comprehensive. We have previously demonstrated that transposon Tn5367 inserts relatively random into the MAP genome (Harris et al., 1999). Another study generated a mutant bank of $5060 \mathrm{~K}-10$ mutants $(P>70 \%)$ (Shin et al., 2006). Based on bioinformatic analysis, 11 mutants were selected for mouse infection experiments (Shin et al., 2006), identifying potential virulence genes $(g c p E$, $p s t A, k d p C, p a p A 2$, impA, umaA1, and fabG2_2). In this study, we constructed an expanded and comprehensive bank of 13,536 K-10 mutants $(P>95 \%)$ and performed phenotypic screens in vitro. We believe this strategy maximizes possible hits in genes important to MAP pathogenesis without relying on studies on other mycobacterial species that may not always translate into similar effects in MAP. We also tested the utility of vectors carrying Tn5370 (McAdam et al., 2002) to generate stable MAP mutants.

\section{MATERIALS AND METHODS \\ BACTERIAL STRAINS, GROWTH CONDITIONS AND RECOMBINANT DNA}

A virulent clinical isolate of MAP (original stock of strain K-10) was used throughout the study (Foley-Thomas et al., 1995). MAP cultures were grown standing with occasional shaking at $37^{\circ} \mathrm{C}$ in complete Middlebrook 7H9 media (MOADC-Plus) as previously described (Harris et al., 1999); see also Section Transposon mutagenesis below. Depending on the experiment, the supplementation with mycobactin J (Allied Monitor, Fayette, MO) varied from 0.5 to $2.0 \mu \mathrm{g} / \mathrm{ml}$. For further characterization of the selected mutants, we used this media without L-tryptophan and casamino acids containing vitamins (MOADC). E. coli $\mathrm{DH} 5 \alpha$ cells, used as cloning hosts, were grown on Luria-Bertani agar or broth supplemented with $50 \mu \mathrm{g} / \mathrm{ml}$ kanamycin (Kan). The conditionally replicating (temperature sensitive) recombinant mycobacteriophage phAE94 (Bardarov et al., 1997) was used to deliver the transposon Tn5367, and signature-tag TM4 phage derivatives of the thermosensitive vector phAE87 were used to deliver Tn5370 (Bardarov et al., 1997; Cox et al., 1999; McAdam et al., 2002). Phage vectors were propagated in M. smegmatis (Msmeg) $\mathrm{mc}^{2} 155$ at $30^{\circ} \mathrm{C}$ as described previously (Bardarov et al., 1997). The Kan-resistance $\left(\operatorname{Kan}^{\mathrm{r}}\right)$ Tn5367 and the hygromycin (Hyg)-resistant $\left(\mathrm{Hyg}^{\mathrm{r}}\right) \mathrm{Tn} 5370$ transposons are derived from the insertion sequence IS1096 from Msmeg (Cirillo et al., 1991).

\section{TRANSPOSON MUTAGENESIS}

MAP cultures were grown to ca. $1.5 \times 10^{8}$ colony forming units $(\mathrm{CFU}) / \mathrm{ml}\left(\mathrm{OD}_{600} 0.38\right.$ to 0.75$)$. Cultures $(50 \mathrm{ml})$ were concentrated by centrifugation and resuspended in $1 \mathrm{ml}$ of $\mathrm{MP}$ buffer (50 mM Tris-HCl, pH 7.6; $150 \mathrm{mM} \mathrm{NaCl;} 2 \mathrm{mM} \mathrm{CaCl} 2$ ). Bacteria and phage were incubated at the non-permissive temperature $\left(37^{\circ} \mathrm{C}\right)$ and stop buffer was added as described previously (Bardarov et al., 1997; Harris et al., 1999). The addition of the stop buffer with sodium citrate helps to prevent further phage infections that may lead to multiple transposon insertions in a single strain. Under these conditions, the potential number of double insertions was shown to be at most 1 in 12 mutants (8.3\%). $\mathrm{Kan}^{\mathrm{r}}$ or $\mathrm{Hyg}^{\mathrm{r}}$ colonies were selected on MOADC-Plus medium without Tween plus $15 \mathrm{~g} / \mathrm{l}$ agar containing $50 \mu \mathrm{g} / \mathrm{ml} \mathrm{Kan}$ or $75 \mu \mathrm{g} / \mathrm{ml} \mathrm{Hyg}$, supplemented with $0.4 \%$ Bacto Casamino Acids (Difco, Becton Dickinson, Franklin Lakes, NJ), and $40 \mu \mathrm{g} / \mathrm{ml} \mathrm{L-}$ tryptophan. Transductants were isolated from these plates after 6-8 week incubation period at $37^{\circ} \mathrm{C}$.

\section{MUTANT SCREENING AND CHARACTERIZATION ASSAYS Visual screen for colony morphology mutants}

To identify colony morphology, MAP mutant strains were grown individually in 96-well microtiter plates in MOADC-Plus fullysupplemented media with $0.5 \mu \mathrm{g} / \mathrm{ml}$ mycobactin $\mathrm{J}$, as described above. Plates were photographed and pictures visually inspected for colony morphology alterations in the bottom of the corresponding wells. Colony morphology alterations were confirmed by further observations on the corresponding agar plates.

\section{Susceptibility to D-cycloserine}

Screen was accomplished by first determining the highest concentration of D-cycloserine (DCS) that would allow wild type growth to occur on MOADC-Plus fully-supplemented solid media (without Tween) for both MAP wild type K-10 and most randomly selected transposon mutants. All mutant strains were then individually replicated onto solid media without and with DCS at the established concentration. After 8 week incubation at $37^{\circ} \mathrm{C}$, DCS susceptibility phenotypes were evaluated and classified as hypersusceptible (failed to grow or grew poorly on DCS), wild type (grew normally, similarly to K-10 on DCS), and resistant (grew faster than K-10 on DCS, reaching a larger colony size).

\section{Miscellaneous screening procedures}

To screen for mutants deficient in siderophore production or secretion, individual mutant colonies were grown on MOADCPlus media, as previously described (Harris et al., 1999). A portion of the bacterial colony was then aseptically transferred onto modified Chrome azurol S (CAS) plates. This media consisted of $72.9 \mathrm{mg} / \mathrm{l}$ of hexadecyltrimethylammonium bromide (Sigma, St Louis, MO), $60.5 \mathrm{mg} / \mathrm{l}$ of CAS (Sigma), $10 \mathrm{ml} / \mathrm{l}$ of iron solution 
( $1 \mathrm{mM} \mathrm{FeCl} \cdot 6 \mathrm{H}_{2} \mathrm{O}, 10 \mathrm{mM} \mathrm{HCl}$ ), $4.7 \mathrm{~g} / \mathrm{l}$ of Middlebrook $7 \mathrm{H} 9$ broth base (Difco, Becton Dickinson), $2 \mathrm{ml} / \mathrm{l}$ glycerol and $15 \mathrm{~g} / \mathrm{l}$ agar adjusted to $\mathrm{pH}$ 5.9. The transferred cell patches were incubated at $37^{\circ} \mathrm{C}$ for 2 weeks and visually screened for the absence of a yellowish halo around the colonies, as previously described (Schwyn and Neilands, 1987). The presence of a halo indicates that siderophores produced and secreted by the colony bind ferric iron in the media.

Biofilm production, thought to be linked to bacterial virulence was also examined. Bacteria were grown in MOADC-Plus broth using plastic tubes and for several weeks. The presence of biofilm was established by visual observation at the sides of the tubes and at the liquid-air interphase. Additional miscellaneous screens are described in the Supplementary Material.

\section{Mutant characterization in BoMac cells}

BoMac (Stabel and Stabel, 1995) monolayers adherent to glass coverslips were infected with approximately the same inoculum (MOI 10:1) in RPMI standard tissue culture medium supplemented with $2 \%$ fetal bovine serum for $2-3 \mathrm{~h}$ (invasion incubation) at $39^{\circ} \mathrm{C}$. Cells were washed, coverslips removed, fixed and acid-fast stained. For each experimental group, a minimum of at least 100 cells were examined at $1000 \mathrm{X}$ magnification under a microscope to determine the percentage of macrophages containing acid-fast bacilli and to enumerate the number of bacteria per macrophage. Bacterial growth at various times post-infection (e.g., Day 0 after invasion incubation and Day 4) was determined by colony counts and BACTEC radiometric assays. Monolayers were lysed with $0.05 \%$ sodium dodecyl sulfate for $30 \mathrm{~min}$, and lysates were inoculated into BACTEC $12 \mathrm{~B}$ vials containing ${ }^{14} \mathrm{C}$ palmitate supplemented with $2.0 \mu \mathrm{g} / \mathrm{ml}$ of mycobactin $\mathrm{J}$ and $1 \mathrm{ml}$ of egg yolk. The BACTEC 460 instrument (Becton Dickinson Diagnostic Instrument Systems, Sparks, MD) was utilized to determine the release of ${ }^{14} \mathrm{CO}_{2}$ from palmitate that was recorded by the instrument as a growth index (recorded every $24 \mathrm{~h}$ until the cumulative index reached 2000 in about 20 days). These indices were converted into viable cell numbers per well (CFUs) using a previously described formula (Lambrecht et al., 1988). The full validation of this method is given elsewhere (Zhao et al., 1997, 1999). As needed, results were confirmed by CFU determinations on MOADC-Plus agar with $2.0 \mu \mathrm{g} / \mathrm{ml}$ mycobactin J.

\section{OLIGONUCLEOTIDE PRIMERS AND PCR}

The oligonucleotide primers used in this study are listed in Table 1. The general conditions for the PCR reactions were: $0.03 \mathrm{ml}$ reaction mixture volume containing $170 \mathrm{nM}$ of each specific primer pair, in the presence of $1 \times \mathrm{NH} 4$-based Reaction Buffer (Bioline USA, Inc., Taunton, MA), $1.7 \mathrm{mM} \mathrm{MgCl}_{2}, 8.3 \%$ (v/v) DMSO, $0.25 \mathrm{mM}$ deoxynucleoside triphosphates and 1.5 units of Biolase ${ }^{\mathrm{TM}}$ DNA Polymerase (Bioline USA, Inc.). Thermo cycler settings of $95^{\circ} \mathrm{C}$ for $5 \mathrm{~min}$, followed by 30 cycles of $95^{\circ} \mathrm{C}$ for $30 \mathrm{~s}, 61^{\circ} \mathrm{C}$ for $45 \mathrm{~s}, 72^{\circ} \mathrm{C}$ for $1 \mathrm{~min}$ and a final extension at $72^{\circ} \mathrm{C}$ for $7 \mathrm{~min}$.

\section{SOUTHERN BLOTTING}

Chromosomal DNA was isolated from wild type and mutant stains. To determine the presence of a transposon insertion,
Table 1 | List of primers used in this study.

\begin{tabular}{ll}
\hline Primer & Sequence $\mathbf{( 5}^{\prime} \mathbf{- 3}^{\prime} \mathbf{)}$ \\
\hline 2E11-IP-F & GCTGCAGCAACCAGCCGA \\
2E11-IP-R & CCACCGTCACCGCAGGTAGA \\
3H4-F & TCGCGGTCCTCGTATTCGCT \\
3H4-R & TGTCGGACGTGTCCGGTCAC \\
4 H2-F & TCAAGTGGGTTGTGCCCGT \\
4 H2-R & GCTACCCAGGAGACGCGCCT \\
22F4-F & GTATCGACCGGTTGTTGATG \\
$22 F 4-R$ & GCCGATGTAGTTGTGGTTGA \\
$40 A 9-I P 1-F$ & GGATGAATCCTCGGCCTTGG \\
$40 A 9-I P 1-R$ & CCGACGGCGTAGTCTGCAAT \\
AMT32 & CTCTTGCTCTTCCGCTTCTTCTCC \\
AMT152 & TTGCTCTTCCGCTTCTTCT \\
Hygro-F & ATAGACGTCGGTGAAGTCGACGAT \\
Hygro-R & GAATCCCTGTTACTTCTCGACCGT \\
IS900-F & GGATGGCCGAAGGAGATTGG \\
IS900-R & GCAGCTCGACTGCGATGTCA \\
IS1096-F & TCGCACTTGACGGTGTA \\
IS1096-R & GTCGGCTCATCGAACAT \\
Kan-F & TCGAGGCCGCGATTAAATTCCACC \\
Kan-R & ATTCATTCGTGATTGCGCCTGAGC \\
MAP1566-F & GCTCTAGAGCTGGCATCAGGGCACTCAAGAAA \\
MAP1566-R & CCCAAGCTTGGGTATTCGCTGCACAGCATGTCAGGT \\
RS6-4 & GTAATACGACTCACTATAGGGCNNNNCATG \\
SP1 & TGCAGCAACGCCAGGTCCACACT \\
SP2 & CTCTTGCTCTTCCGCTTCTTCTCC \\
STM5370-1 & TGCTAGGCGTCGGCCATTAGC \\
T7 & TAATACGACTCACTATAGGG \\
&
\end{tabular}

for mutants derived from Tn5367, DNA samples were digested with EcoRI, which does not cut within this transposon. For Tn5370 derivatives, DNA samples were digested with NheI and NdeI, which do not cut within this transposon. Digested DNA fragments from both wild type and mutant strains were electrophoresed on a standard agarose gel and transferred to a nylon membrane (Osmonics, Inc., GE Water and Process Technologies, Trevose, PA). Probes were prepared by PCRamplification using specific primers (Table 1): IS1096 probe was amplified from Msmeg genomic DNA with IS1096-F and IS1096-R and IS900 probe was amplified from MAP genomic DNA using IS900-F and IS900-R. The Kan-resistant aph gene probe for Tn5367 was amplified from pMV262 (Connell et al., 1993), or plasmids derived there from, with Kan-F and KanR. The Hyg-resistant hyg gene probe for Tn5370 was amplified from pYUB854 (Bardarov et al., 2002), or plasmids derived there from, using Hygro-F and Hygro-R. Probes were labeled with $\alpha-[32 \mathrm{P}]-\mathrm{dCTP}(3000 \mathrm{Ci} / \mathrm{mmol})$ using the Rediprime DNA Labeling System (Amersham-Pharmacia Biotech, Inc., Molecular Dynamics Div., Piscataway, NJ) as described by the manufacturer. Hybridization and detection were done as described previously with minor modifications (Feng et al., 2002). Southern blot images were digitally captured and processed only to enhance brightness and contrast (Bio-Rad Imaging system, Hercules, CA). 


\section{SEOUENCING OF THE TRANSPOSON-INSERTION SITE}

In order to locate the transposon insertion sites in the $\mathrm{K}$ 10 genome, DNA was amplified for sequencing analysis by a nested PCR as previously described (McAdam et al., 2002). A new primer designated STM5370-1, common to both transposons, was used in place of the original SP1 primer due to differences in the Tn5367 and Tn5370 transposon sequences. This primer was designed based on sequence information of Tn5370 provided Dr. Jeffrey Cirillo (Texas A\&M Health Science Center) that was further supplemented by our own sequencing studies. The first PCR used the following primers: RS6-4 (degenerate) and STM5370-1. The variable size PCR products $(1 \mu \mathrm{l})$ from the first cycle were used in a second PCR cycle with primers $\mathrm{T} 7$ and SP2 that are internal to the first cycle PCR product. These final PCR products were purified by Wizard PCR Preps DNA Purification System (Promega, Madison, WI). The samples were run on $1.5 \%$ agarose gel electrophoresis to recover the most concentrated DNA bands and purified by the GeneClean ${ }^{\circledR}$ III Kit (Qbiogene, MP Biomedicals, LLC, Solon, $\mathrm{OH})$. DNA samples were sent to The University of Nebraska Genomics Core Research Facility for sequencing, using AMT152 as primer (Shin et al., 2006). Sample runs were performed on a Beckman-Coulter CEQ8000 or CEQ2000XL 8-capillary DNA sequencer using dye-terminator chemistry to provide $500-650 \mathrm{bp}$ of sequence.

\section{GROWTH CURVES IN BROTH CULTURES}

MAP strains were grown to late exponential phase $\left(\mathrm{OD}_{600}\right.$ between 1.0 and 1.5 ) at $37^{\circ} \mathrm{C}$ from glycerol stocks in MOADC media $(1.0 \mu \mathrm{g} / \mathrm{ml}$ mycobactin $\mathrm{J})$, appropriately supplemented with antibiotics as needed. Applicable culture volumes were inoculated into $45 \mathrm{ml}$ of MOADC media in T75 flasks with vented caps so that the initial $\mathrm{OD}_{600}$ was approximately 0.05 . Each culture was incubated at $37^{\circ} \mathrm{C}$ standing with $\mathrm{OD}_{600}$ readings being taken on days $0,3,5,7,10,12,14,17,21,24$, and 28 around the same time each day. CFU counts were also determined on MOADC agar.

\section{PRIMARY BOVINE MACROPHAGE ASSAY}

Monocyte-derived macrophages (MDMs) from a JD negative dairy cow were elutriated and enriched as described (Lamont and Sreevatsan, 2010). Incubations were conducted in a $37^{\circ} \mathrm{C}$ humidified chamber containing $5 \% \mathrm{CO}_{2}$. Approximately $2.0 \times$ $10^{7}$ MDMs were seeded into separate T25 flasks and allowed to adhere for $2 \mathrm{~h}$. MDMs were washed thrice to remove nonadherent cells and fresh RPMI 1640 supplemented with 2\% autologous serum was added prior to MAP infection. Subcultured MAP (K-10 wild type or mutant strains) were grown to an $\mathrm{OD}_{600}$ of 0.5 (approximately $1.0 \times 10^{8}$ cells $/ \mathrm{ml}$ ) and pelleted at $8000 \times \mathrm{g}$ for $10 \mathrm{~min}$. The pellet was washed thrice using $1 \mathrm{X}$ phosphate buffered saline (PBS), resuspended in RPMI 1640 containing 2\% autologous serum (MOI 10:1), and vortexed for $5 \mathrm{~min}$. MAP cells were repeatedly drawn through a 21 -gage needle to break bacterial clumps. Cells were incubated at $37^{\circ} \mathrm{C}$ for $5 \mathrm{~min}$ to sediment any remaining clumps and only the upper $2 / 3$ rd volume was used for infection. MDMs were infected with MAP for $2 \mathrm{~h}$ (invasion incubation), washed thrice with $1 \mathrm{X}$ PBS to remove nonadherent bacteria, and recovered in RPMI 1640 supplemented with $2 \%$ autologous serum for the following post-infection time points: $0,2,6,12,24$, and $48 \mathrm{~h}$ after the completion of the invasion incubation period. Upon completion of each post-infection time point, MDMs were washed thrice in 1X PBS and incubated with $0.01 \%$ Triton $\mathrm{X}-100$ in PBS for $5 \mathrm{~min}$ at room temperature. The lysate was subjected to differential centrifugation at $388 \times \mathrm{g}$ and $8000 \times \mathrm{g}$ for $5 \mathrm{~min}$ each to remove MDMs and pellet bacteria, respectively. MAP pellets were washed thrice in $1 \mathrm{X}$ PBS at $8000 \times \mathrm{g}$ for $5 \mathrm{~min}$ to remove any remaining detergent, and resuspended in $1.0 \mathrm{ml}$ of $1 \mathrm{X}$ PBS. All time points were assayed in triplicate. MAP inoculum and post-infection time points were separately stained using the Baclight Live/Dead kit (Invitrogen, Carlsbad, CA) and gated for live cells on a flow cytometer per manufacturer's instructions as described (Lamont and Sreevatsan, 2010).

\section{CALCULATION OF GROWTH PARAMETERS AND STATISTICAL ANALYSIS}

Statistical analyses to determine $P$-values presented in the text and figures were performed using the Statistical Analysis System (SAS) software version 9.1.3, 2004 (SAS Institute) using the Mixed Procedure Subroutine. Post-hoc comparisons were carried out using Tukey adjusted $P$-values. For calculations involving ODs and CFUs, logarithmic transformations were applied.

\section{NUCLEOTIDE SEQUENCE ACCESSION NUMBERS}

The DNA sequences of transposons Tn5367 (Cirillo, J. D., McAdam, R. A., Weisbrod, T. R., Barletta, R. G. and Jacobs, W. R. Jr.) and Tn5370 (Cirillo, J. D., Rathnaiah, G., McAdam, R. A., Weisbrod, T. R., Barletta, R. G. and Jacobs, W. R. Jr.) have been assigned GenBank accession numbers KM232614 and KM232615, respectively.

\section{RESULTS}

\section{GENERATION AND SCREENING OF A TRANSPOSON MUTANT BANK}

To investigate virulence factors of MAP, a transposon mutant bank of MAP wild type K-10 was constructed. The transposon Tn5367 carrying a Kan resistance marker (Bardarov et al., 1997) randomly inserted into the bacterial chromosome by transposition from a thermosensitive phage delivery vector. Nine independent infections ( 8 with phage and 1 control with no phage) were carried out to ensure that sibling mutants were minimally represented. A total of 13,536 Kan-resistant mutants were collected and stored individually in an indexed collection of 141 96-well plates stored at $-80^{\circ} \mathrm{C}$. Each mutant is identified by the plate number and the coordinates of the well (a letter for the row and a number for the column): e.g., mutant $4 \mathrm{H} 2$ is stocked in plate 4 and the well that intersects row $\mathrm{H}$ and column 2 in a 96-well microtiter plate. In addition, to facilitate screening procedures, pools of 96 or 24 mutants were also stocked so they could be easily referred to individual mutants in the indexed collection. Assuming random transposition and 4500 target genes with single transposon insertions $[\ln (1-P)=N \times \ln (1-1 / 4500)]$, this number of mutants yields a $P$-value of approximately $95 \%$ for insertions in non-essential genes. The full library or a subset of strains were subjected to various individual screens to find strains of interest for studying MAP physiology and pathogenesis, and 
principally to search for attenuated strains that could be used as first-generation vaccine candidates in animal trials. A set of 123 strains, fairly well distributed through the various plates, were analyzed to show that the library was composed of strains with diverse phenotypes (Table S1).

\section{Visual screen for colony morphology mutants}

Colony morphology of microorganisms is well known to influence virulence, drug susceptibility and macrophage survival (Reddy et al., 1996). To identify colony morphology of MAP mutants, each of the 13,536 mutant strains was grown on MOADC-Plus media, visually observed and photographed with a camera. Using this procedure, eight mutants were found that exhibited colony morphologies different from the wild type strain (1F3, 4H2, 16B11, 22F4, 65E9, 69D12, 73D7, and 84D12). In particular, $4 \mathrm{H} 2$ displayed highly irregular colony borders and a rougher surface appearance as compared to K-10 (Figure 1 and Figure S1A). In contrast, mutant 22F4 displayed a smooth colony morphotype (Figure S1C). These two mutant strains were further tested in BoMac cells (see Section Miscellaneous screening). It is noted that visual screens may depend on the media used and undoubtedly have a degree of subjectivity. Thus, less obvious but yet relevant differences in colony morphotype may not have been identified.

\section{Susceptibility to DCS}

DCS inhibits peptidoglycan biosynthesis in mycobacteria by the combined inhibition of D-alanine racemase (Alr) and Dalanine ligase (Chacon et al., 2002, 2009; Feng and Barletta, 2003; Halouska et al., 2014). Inactivation of the Msmeg alr gene results in a 500-fold reduction in the ability of Msmeg to survive within phagocytic cells and also determines a DCS hypersusceptible phenotype (Chacon et al., 2009). In addition, there are several potential alterations that can lead to DCS susceptibility or resistance including alterations of surface proteins that may also play a role in pathogenesis. To test this hypothesis in MAP, the entire mutant bank was screened for DCS susceptibility. First, it was determined that $20 \mu \mathrm{g} / \mathrm{ml}$ DCS was the maximum concentration

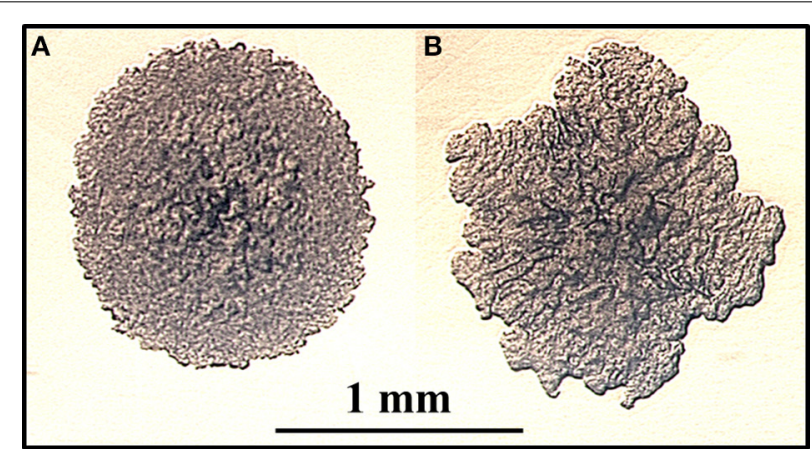

FIGURE 1 | (A,B) Identification of a MAP mutant with altered colony morphology. MAP strains were grown on fully supplemented MOADC-Plus medium until colonies were approximately $1 \mathrm{~mm}$ in diameter (see scale bar) and photographed at $100 \mathrm{X}$ magnification. Colony morphologies for the wild type strain K-10 (A) and the Tn5367 transposon mutant $4 \mathrm{H} 2$ (B) are displayed. that had no effect on the growth of K-10 and a subset of randomly picked transposon mutant strains. Using this approach, 98 mutant strains were found hypersusceptible to DCS. Sixty-three strains did not grow: e.g., colony morphotypes 1F3, 16B1, and 22F4 (Figures S1C,D); and normal colony morphotypes 12G8, 37D1, and 30H9. Thirty-five mutants grew poorly: e.g., normal colony morphotypes 3D4 and 23B5, but displayed normal growth without the drug. However, 15 DCS resistant mutants grew faster, as evidenced by expanded colony sizes: e.g., colony morphotypes 4H2 (Figures S1A,B), 65E9 and 69D12; and normal colony morphotype 40A9. The colony morphotypes $4 \mathrm{H} 2$ and 22F4 (see Section Visual screen for colony morphology mutants), as well as strains $30 \mathrm{H} 9$ and $40 \mathrm{~A} 9$, were selected for further testing in BoMac cells (see Section Miscellaneous screening). In contrast, colony morphology mutants 73D7 and 84D12 displayed wild type susceptibility. In summary, this screen yielded 113 potentially attenuated strains, the greatest number among the assays performed.

\section{Miscellaneous screening}

Chromogenic screening of the entire mutant bank was performed to identify mutants unable to synthesize and/or secrete siderophores other than mycobactin. Since MAP is a known auxotroph for mycobactin, the goal of this experiment was to test the hypothesis that mycobacterial mutants unable to synthesize and/or secrete any other type of siderophore may be attenuated (Fiss et al., 1994). This method identified mutant 1F3 that grew significantly slower than K-10 in standard mycobactin J supplemented media did not produce a halo on the modified CAS medium plates (Figure 2). This phenotype suggests an impairment of the synthesis or transport of an alternative siderophore different from mycobactin. This mutant also displayed colony morphology alterations (see Section Visual screen for colony morphology mutants) and DCS hypersusceptibility (see Section Susceptibility to DCS). As stated in Section Generation and screening of a transposon mutant bank, the entire mutant bank, as well as 1F3, was stocked as a 96 well-plate culture. Replicate plating on MOADC-Plus for the CAS assay was conducted 6 months thereafter with all mutants, except 75, being viable. However, in a subsequent replication years later, mutant 1F3 grew poorly but in sufficient amounts to conduct a preliminary goat experiment when sent to collaborators in Israel (Livneh et al., 2005). Unfortunately, subcultures at both laboratories no longer grew.

Biofilm screening was also performed with a limited set of ca. 300 mutants to identify strains deficient in biofilm production (Figure S2). We identified mutants 2E11, 4E1, and 4E7 that were impaired in biofilm production. However, mutant 2E11 biofilm deficiency was due to poor growth in the assay. In contrast, mutant $4 \mathrm{~F} 3$ produced more biofilm than the wild type strain. Additional screens identified mutants 3D4 and 3B11 with impaired cell association in BoMac cells (Figure S3), and strain 3D9 and 4E7 displayed impaired clump formation (Figure S4). All of these seven mutants did not display altered colony morphotypes. In addition, all of these mutants, except for 3D4, had normal DCS susceptibilities. Nonetheless, serendipitously, mutant 2E11 was selected for further studies as preliminary 


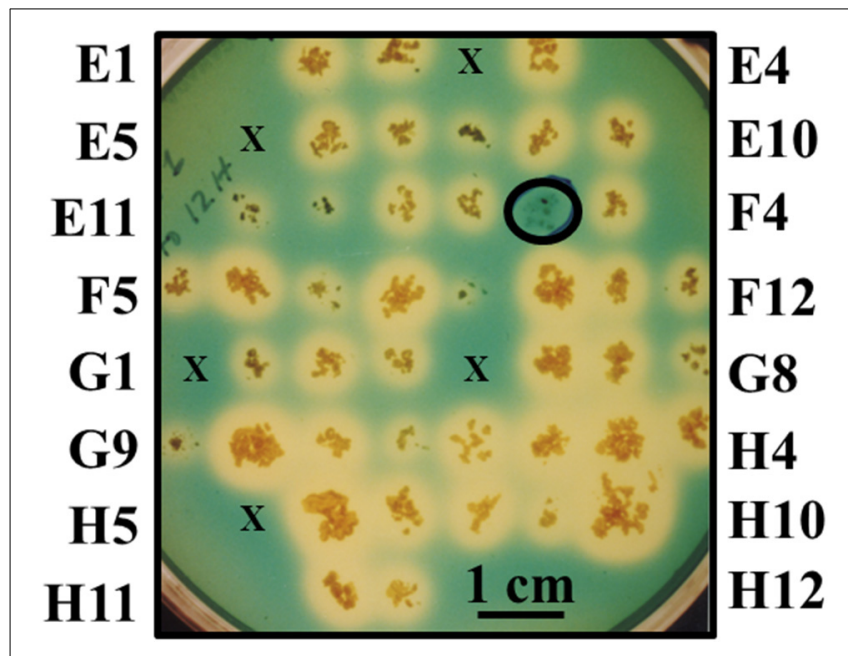

FIGURE 2 | Direct visual screen for MAP mutants unable to produce or secrete siderophores. Mutants were grown on MOADC-Plus media for about 8 weeks, transferring a portion of the colony to Chrome azurol S plates since this media does not allow for growth. Labels for the outermost colonies are shown for each row and column (according to 96-well plate nomenclature) to designate individual mutants. Mutants that failed to grow initially are represented by an " $x$ " and mutants that produced a halo are controls. A scale bar is provided to estimate halo size (blue to yellow color change when a strong chelating agent removes iron from the dye) after 2 weeks of incubation at $37^{\circ} \mathrm{C}$. The circled colony without a halo is mutant 1F3 impaired in siderophore production or secretion.

assays with MDMs indicated significant attenuation (see Section Interaction of wild type and mutant strains with primary bovine macrophages).

\section{MAP mutant characterization in BoMac cells}

The invasion, survival and/or replication of selected MAP strains were first tested in the BoMac cell line, as these cells provide reproducibility over primary macrophages for the extended period of time that would be required to test a large number of mutants. Preliminary assessment of bacillary counts for representative strains was performed by microscopic examination of acid-fast stained monolayers on glass coverslips. Results were confirmed by CFU determination and BACTEC assays that were better suited for screening assays. Finally, the more promising mutants were screened in this fashion by BACTEC assays. Representative results are shown for two sets of independent experiments (Figure 3).

In the first set (Figure 3, left), K-10 was compared with 3H4 (with normal colony morphology, but unable to grow in DCS), $4 \mathrm{H} 2$ and 22F4. Results at Day 0 (after invasion incubation) showed that the invasiveness of $3 \mathrm{H} 4$ and $22 \mathrm{~F} 4$ were similar to $\mathrm{K}-10(P>0.1)$, but $4 \mathrm{H} 2$ was significantly less invasive than the wild type strain $\mathrm{K}-10(P<0.01)$, and also less invasive than $3 \mathrm{H} 4$ $(P<0.01)$ and $22 \mathrm{~F} 4(P<0.05)$. Survivability for each strain was estimated by comparing the CFUs at Day 0 and Day 4: interaction of K-10 with BoMac cells resulted in decreased survival but the effect was not statistically significant $(P>0.1)$, reduced survivability was more pronounced and approached significance for 3H4 $(P=0.055)$, there was almost no change for $4 \mathrm{H} 2(P>0.1)$, but reduction in CFUs was significant for 22F4

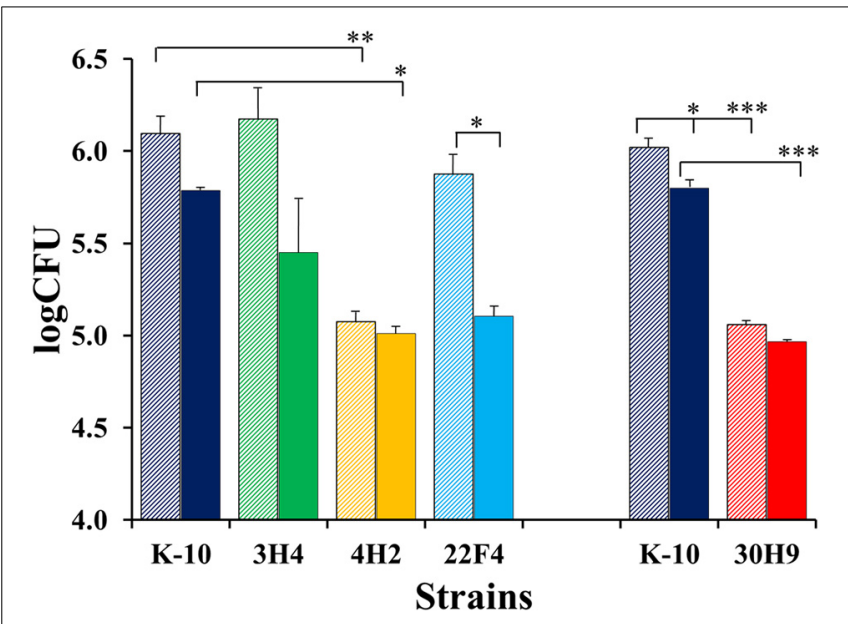

FIGURE 3 | Interaction of MAP wild type K-10 and mutant strains with the BoMac cell line. Macrophage monolayers were infected with bacteria at a multiplicity of 10:1, incubated and lysed. Counts were taken at Day 0 (after invasion; stripe bars) and Day 4 (post-infection; solid bars). Bacilli were enumerated using the BACTEC radiometric assay system by recording growth indices every $24 \mathrm{~h}$ until this cumulative index reached 2000 (usually 20 days). These indices were converted into viable cell numbers per well (CFUs) using a previously described formula that has been extensively validated for MAP (Lambrecht et al., 1988; Zhao et al., 1997, 1999). Results represent means $(n=2) \pm$ standard error of the mean. Statistical significance for pairwise determinations displayed by a line with tick marks is denoted with asterisks: ${ }^{*} P$-value $<0.05,{ }^{*} P$-value $<0.01$ and *** $P$-value $<0.001$

$(P<0.05)$. Comparison of the overall macrophage CFUs at Day 4 among all strains indicated that the $3 \mathrm{H} 4$ burden was not significantly different from K-10 $(P>0.1)$, while 22F4 showed a reduced burden but was not significantly different $(P=0.075)$, while $4 \mathrm{H} 2$ showed significantly less CFUs $(P=0.039)$.

Results for the second set (Figure 3, right), indicated that $30 \mathrm{H} 9$ was less invasive than $\mathrm{K}-10(P<0.001)$, but showed no significant difference $(P>0.1)$ in survivability from Day 0 to Day 4 . In addition, 30H9 had a significantly lesser CFU burden than K-10 at Day $4(P<0.001)$. Notably, for this set, K-10 displayed a similar reduction in survivability from Day 0 to Day 4, but in this case, on account of more precise measurements and reduced number of means comparisons, the decrease was statistically significant $(P<0.05)$. In a separate assay, mutants $12 \mathrm{G} 8$ and 37D1 were also shown to be attenuated in BoMac cells. Mutant 23B5 displayed an attenuated phenotype when directly tested in MDMs infection assays (Table $\mathrm{S} 1$ ).

In summary, $4 \mathrm{H} 2$ and $30 \mathrm{H} 9$ were less invasive than the wild type K-10 strain, but had similar intracellular survival as K-10. In contrast, 22F4 was the only strain that was significantly less able to survive, by the end point, at Day 4 post-infection. The combined effects of invasion and survival, as indicated by the absolute CFU burdens as determined by the BACTEC assay, at Day 4, indicated that $4 \mathrm{H} 2$ and $30 \mathrm{H} 9$ were definitely attenuated in BoMac cells, predominantly by their reduced invasiveness. Thus, these two strains, once internalized at lower CFU burdens, persisted post-infection at approximately constant numbers. In contrast, 22F4 was attenuated because of its reduced survival, but the effect 
was of lesser degree. The normal colony morphotype mutant 3H4 behaved similarly to the wild type strain K-10.

\section{FURTHER CHARACTERIZATION OF TRANSPOSON MUTANTS OF INTEREST}

To further characterize mutants of interest, Southern hybridization analyses, and identification of transposon insertion sites by nested PCR sequencing were performed. Tests to identify specific transposon mutants in mixed infections were developed. In addition, these mutants were characterized by determination of growth curves in broth cultures and their interactions with primary bovine macrophages.

\section{Southern hybridization}

To characterize the molecular events that gave rise to the mutant strains, Southern hybridizations were performed with various probes. Hybridization with a probe specific for the common transposon insertion sequence IS1096 showed unique size bands for 33 mutant strains (not all positive strains shown in Figure 4; see Table S1), indicating the presence of a single copy of the corresponding transposon (Figures $4 \mathbf{A}-\mathbf{B}$ ). These results were confirmed by hybridization with a probe specific for either the Kan-resistant aph marker for Tn5367 derivatives, or the Hygresistant hyg marker for Tn5370 derivatives. Both probes yielded bands in the same gel positions as the IS1096 probe (unpublished results). To verify that mutant strains were derived from K-10 and that no major molecular rearrangements have occurred, a probe specific for IS900 was used. As expected, this analysis showed that all mutant strains analyzed had the same hybridization banding pattern as the wild type strain K-10 (Figures 4C-D). As mutants were directly analyzed from the original stocks (Figure 4A), we noted that 22F4 did not show a clear hybridization band with IS1096, and mutant 40A9 showed a very faint band. Upon replication to make large stocks for further analyses, mutant 22F4 clearly displayed the corresponding hybridization band, but 40A9 displayed no band, indicating the potential loss of the transposon insertion (Figure 4B). In addition, the original stock of mutant 5E5 did not display a hybridization band.

\section{Transposon insertion site identification by sequencing of nested PCR amplicons}

The genomic regions containing transposon insertions of interest were amplified by nested PCR and sequenced as described (see Section Sequencing of the transposon-insertion site). Transposon insertion sites were identified by performing BLASTN identity searches of the MAP DNA sequences adjacent to transposon insertion sites against the K-10 complete genome sequence. In mutants 22F4, 30H9 and STM68, the transposon inserted within genes of interest (Figure 5A). In mutant 22F4, the transposon insertion site was mapped internal to MAP0460 (lsr2) and located $110 \mathrm{bp}$ from the ATG start codon. For mutant $30 \mathrm{H} 9$, transposon insertion sites were mapped as internal to MAP1566.

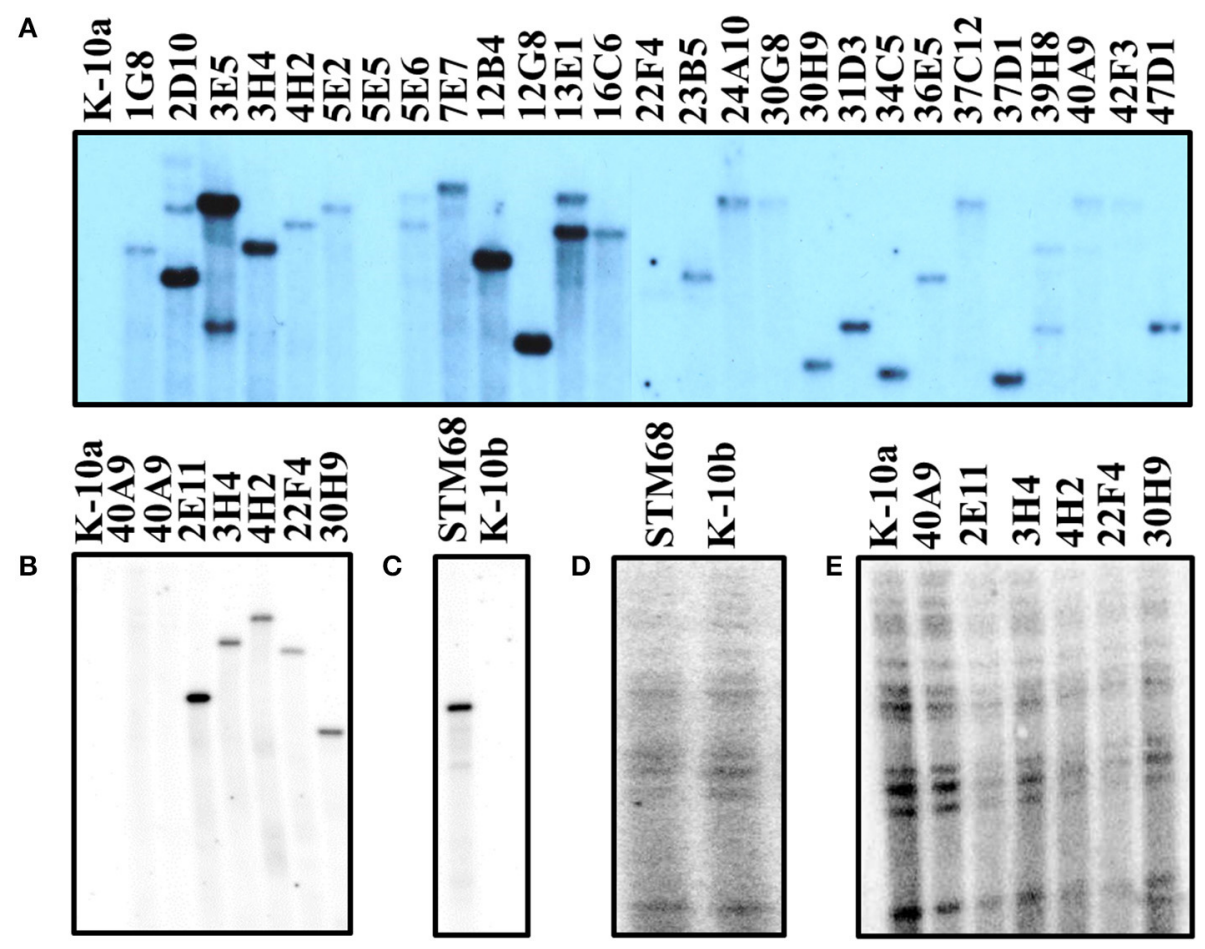

FIGURE 4 | (A-E) Southern blot analysis of MAP wild type K-10 and transposon insertion mutants. DNA samples from Tn5367 mutants and the wild type strain (K-10a) were digested with EcoRI (A,B,E). DNA samples from the Tn5370 mutant STM68 and the wild type strain (K-10b) were digested with $\mathrm{Nhel}$ and $\mathrm{Ndel}(\mathbf{C}, \mathbf{D})$. DNA samples were run on agarose gels, transferred to a nylon membrane and hybridized with the IS 1096 probe to verify the presence of the transposon (A-C) and the IS900 probe to confirm that the mutants were derived from MAP (C,D). (B-E) are hybridization images generated from the same Southern transfer, while (A) was from an independent transfer. 


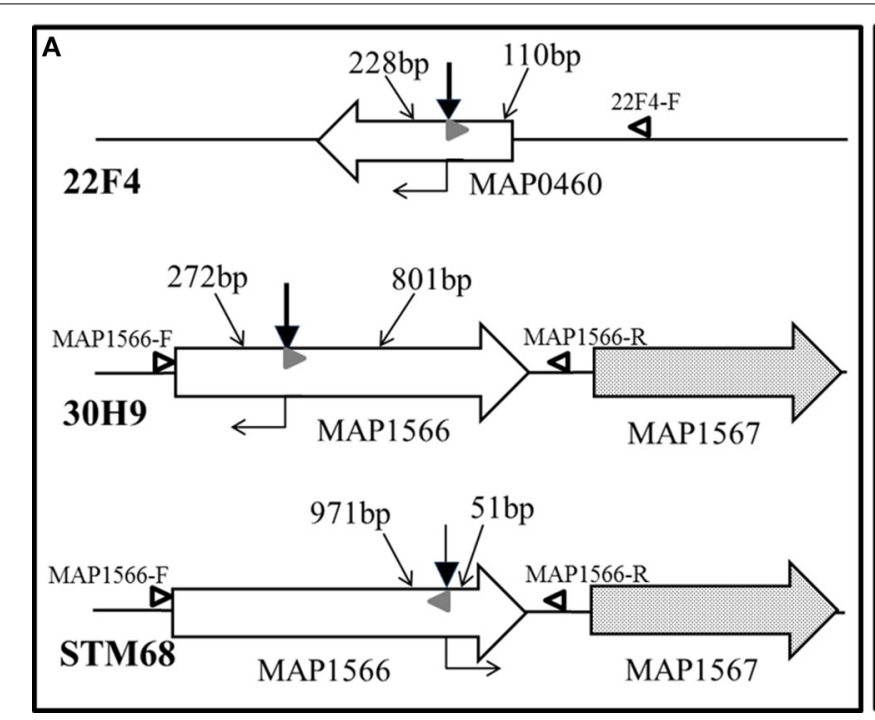

FIGURE 5 | (A,B) Transposon insertion sites of MAP mutants. Solid line wide arrows represent ORFs and indicate the direction of transcription. Dotted fill wide arrows exemplify ORFs subjected to a potential polar effect due to the transposon insertion. The dashed line wide arrow represents a gene cluster transcribed in the same direction. Bold thick vertical arrows stand for Tn5367 while the bold thin arrow symbolizes Tn5370 insertion points. Regular thin arrows point to the DNA segments indicating distances (bp) between the transposon

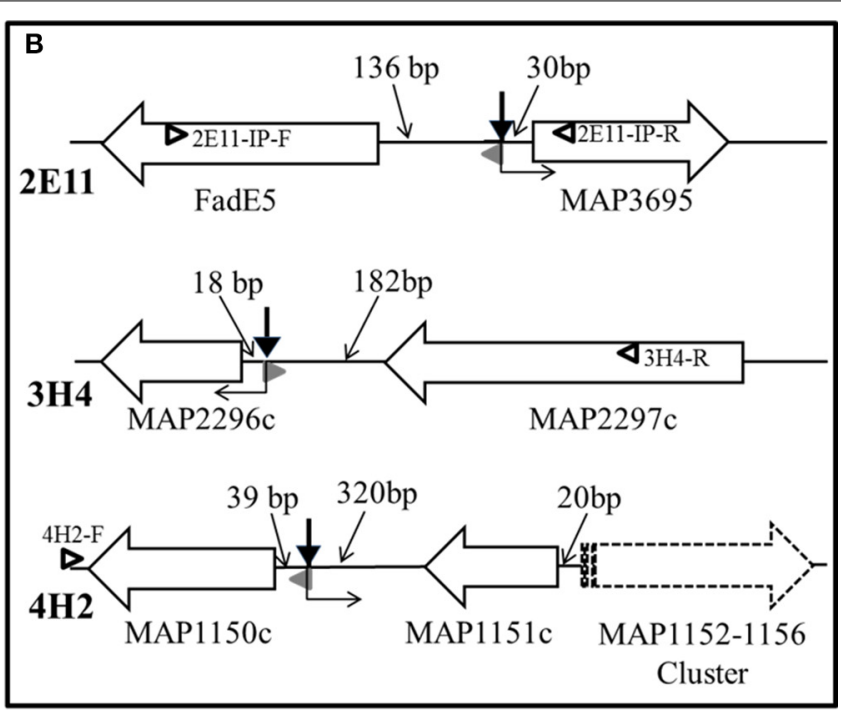

insertion point and the start or end of the corresponding ORF. Open black and solid gray (AMT32) sideway triangles represent primers used in PCR mutant identifications for mixed infection experiments (see Figure 6). Right angle bent arrows indicate the direction of transcription of the drug selection markers (aph in Tn5367 derivatives; hyg in Tn5370 derivative STM68) in the transposon. Diagram representing relevant genomic regions for mutants are represented: (A) 22F4, 30H9, and STM68; and (B) 2E11, 3H4, and $4 \mathrm{H} 2$.
Serendipitously, another screen from a signature-tagged mutagenesis experiment (ca. 300 mutants) identified a Tn5370 mutant (STM68) with an insertion in the same gene as in $30 \mathrm{H} 9$, but the insertion in STM68 was mapped toward the $3^{\prime}$-end coding sequence of MAP1566. STM68 is a stable mutant because Tn5370 lacks the transposase within the transposed region avoiding the potential for further rearrangements in the mutant strain. In $30 \mathrm{H} 9$, Tn5367 is located 272 bp from the ATG start codon, while mutant STM68 carries a Tn5370 insertion $51 \mathrm{bp}$ from the TAA termination codon. Thus, for STM68, the insertion may also exert a polar effect on MAP1567 located downstream within the operon. Likewise, though the insertion in $30 \mathrm{H} 9$ is well upstream within the MAP1566 coding sequence, a polar effect on MAP1567 cannot be ruled out.

In mutants $2 \mathrm{E} 11,3 \mathrm{H} 4$, and $4 \mathrm{H} 2$, the transposon Tn5367 inserted in the intergenic regions (Figure 5B). For mutant 2E11, Tn5367 inserted $130 \mathrm{bp}$ upstream from FadE5 and $30 \mathrm{bp}$ upstream from MAP3695. In 3H4, the insertion site was 18 bp upstream from MAP2296c and 182 bp downstream from MAP2297c. In 4H2, the transposon insertion was 39 bp upstream from MAP1150c and $320 \mathrm{bp}$ downstream from MAP1151c. In all these cases, it is hypothesized that the transposon affects the expression of either or both genes adjacent to the insertion sites, or other genes in close proximity. Sequencing of the original isolate of 40A9 putatively identified a transposon insertion site in the intergenic region between MAP0282c and MAP0283c. However, as this insertion point could not be further verified and 7 consecutive nucleotides of primer STM5370-1 matched within MAP0282c sequence, the possibility of spurious priming could not be ruled out. Comparison of the full genomic sequence of 40A9 with K-10 may be necessary to identify any sequence change in this mutant strain. However, this analysis is beyond the scope of this work.

\section{Polymerase chain reaction for mutant verification}

To demonstrate the presence of specific transposon insertion mutants in mixed infections, such as protection challenge experiments, it was necessary to develop a specific PCR test for each mutant strain. The test is also confirmatory of the sequencing studies to rule out any spurious transposon insertion site identifications. To accomplish this goal, based on the sequencing results, amplicons were synthesized with primers specific for the sequences adjacent to the transposon insertion sites (Table 1), and analyzed on agarose gels (Figure 6). Amplification of wild type K-10 DNA with primers MAP1566-F (within MAP1566 but upstream from the insertion point) and MAP1566-R (within the intergenic region between MAP1566 and MAP1567) resulted in a DNA fragment of $1179 \mathrm{bp}$ (Lanes 3 and 7); these primers yielded fragments of $3474 \mathrm{bp}$ (Lane 4) and $4560 \mathrm{bp}$ (Lane 8), respectively for mutants STM68 and 30H9. These sizes are as expected for the insertion of $\operatorname{Tn} 5370$ (2295 bp) or Tn5367 (3381 bp), respectively. Likewise, amplification of K-10 (Lane 5) and 2E11 (Lane 6) with primers 2E11-IP-F (within MAP3695) and 2E11-IP-R (within FadE5) resulted in a DNA fragment of $1845 \mathrm{bp}$ and $5226 \mathrm{bp}$, respectively; thus confirming the predicted insertion of Tn5367 in the FadE5 and MAP3695 intergenic region.

PCR of mutants $3 \mathrm{H} 4,4 \mathrm{H} 2$, and $22 \mathrm{~F} 4$ with specific primers (3H4-F, 3H4-R, 4H2-F, 4H2-R, 22F4-F, and 22F4-R, respectively) 
from regions adjacent to the insertion gave the same amplification product to that of K-10 (unpublished results). However, we were able to confirm the insertion point using a specific primer for the adjacent sequences and primer AMT32 specific for both Tn5367 and Tn5370 (McAdam et al., 2002; Shin et al., 2006). In this case, $\mathrm{K}-10$ gave no amplification products (unpublished results). All of the mutant strains gave amplification products of the expected sizes: STM68 (1160 bp, Lane 10), 2E11 (1890 bp, Lane 11), 3H4 (1974 bp, Lane 12), 4H2 (1431 bp, Lane 13), 22F4 (561 bp, Lane 14), and $30 \mathrm{H} 9$ (941 bp, Lane 15). We obtained the same amplification product for K-10 and 40A9 using primers 40A9-IP1-F and

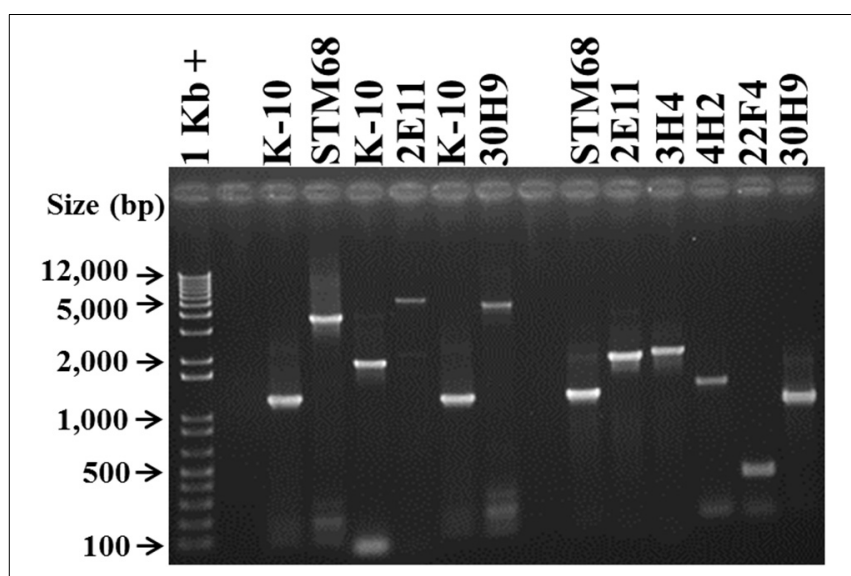

FIGURE 6 | Analysis of MAP Tn5367 (3,381 bp) and Tn5370 (2295 bp) insertion mutants by PCR. Lane 1, Invitrogen TrackIt ${ }^{\mathrm{TM}} 1 \mathrm{~Kb}$ Plus DNA Ladder; Lanes 2 and 9, empty. Amplicons for the DNA template-primer combinations and corresponding sizes are: Lanes 3 and 7, K-10 with MAP1566-F and MAP1566-R, $1179 \mathrm{bp}$; Lane 4, STM68 with MAP1566-F and MAP1566-R, 3474 bp; Lane 5, K-10 with 2E11-IP-F and 2E11-IP-R, 1845 bp; Lane 6, 2E11 with 2E11-IP-F and 2E11-IP-R, 5226 bp; Lane 8, 30H9 MAP1566-F and MAP1566-R, $4560 \mathrm{bp}$; Lane 10, STM68 with MAP1566-F and AMT32, $1160 \mathrm{bp}$; Lane 11, 2E11 with 2E11-IP-F and AMT32, $1890 \mathrm{bp}$; Lane 12, 3H4 with 3H4-R and AMT32, 1974 bp; Lane 13, 4H2 with 4H2-F and AMT32, $1431 \mathrm{bp}$; Lane 14, 22F4 with 22F4-F and AMT32, 561 bp; Lane 15, 30H9 with MAP1566-R and AMT32, $941 \mathrm{bp}$. Relevant band sizes (bp) for the DNA ladder are displayed to the left.

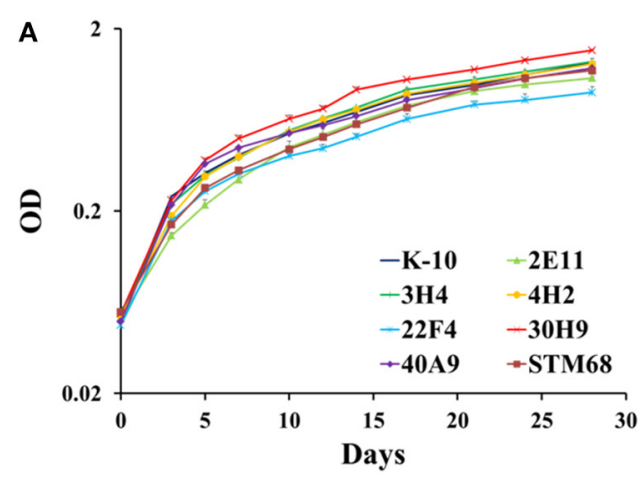

FIGURE 7 | (A,B) Growth curves of MAP wild type K-10 and mutant strains. Strains were inoculated into MOADC media at an initial optical density $\left(O D_{600}\right)$ of approximately 0.05 . Cultures were incubated at $37^{\circ} \mathrm{C}$
40A9-IP1-R adjacent to MAP0283c and MAP0282c (unpublished results). Thus, results confirmed the location of the transposon insertion sites obtained by DNA sequencing. Furthermore, the unique sizes of the PCR amplicons were successfully used to identify the presence of each mutant strain in animal experiments (Bannantine et al., 2014; Hines et al., 2014).

\section{Determination of growth curves in broth cultures}

As these MAP mutants were developed as first generation candidate vaccine strains, it was important to determine if their corresponding in vitro growth rates in broth cultures were similar to the wild type K-10 strain. Determination and statistical analysis of growth curves indicated that both wild type and mutant strains grew approximately at the same rate in MOADC, as assessed by $\mathrm{OD}$ (Figure 7A) or CFU (Figure 7B) determinations. In particular for OD values, growth rates of the mutant strains were not significantly different compared to $\mathrm{K}-10(P>0.05)$, except for $30 \mathrm{H} 9$ that grew slightly faster $(P=0.047)$. Likewise the more detailed CFU calculations showed a similar effect, but in this case $3 \mathrm{H} 4$ grew faster $(P=0.021), 22 \mathrm{~F} 4$ had a slower rate $(P=0.019)$ and growth of $30 \mathrm{H} 9$ did not differ significantly $(P>0.05)$ from $\mathrm{K}-10$. These results suggest that the mutations in these strains are related to properties that relate to virulence such as survival and replication in BoMac cells rather than standard physiological traits. This characteristic is desirable for candidate live-attenuated vaccine strains as the isolates can be readily propagated in broth cultures to prepare inoculums for vaccine delivery.

\section{Interaction of wild type and mutant strains with primary bovine macrophages}

As macrophages play a key role in MAP infections, to confirm the results obtained with BoMac cells and characterize additional mutants, we determined the interaction of wild type and mutant strains with primary macrophages. MDMs were infected with each strain at a MOI of 10:1, incubated to allow invasion, washed and lysed immediately or at various time points post-infection to determine colony counts (Figures 8-10). The results indicated that strains $2 \mathrm{E} 11(P<0.05), 3 \mathrm{H} 4(P<0.01)$ and 22F4 $(P<0.001)$ were significantly less invasive than $\mathrm{K}-10$ (Figure 8). No major differences were observed for all four cases

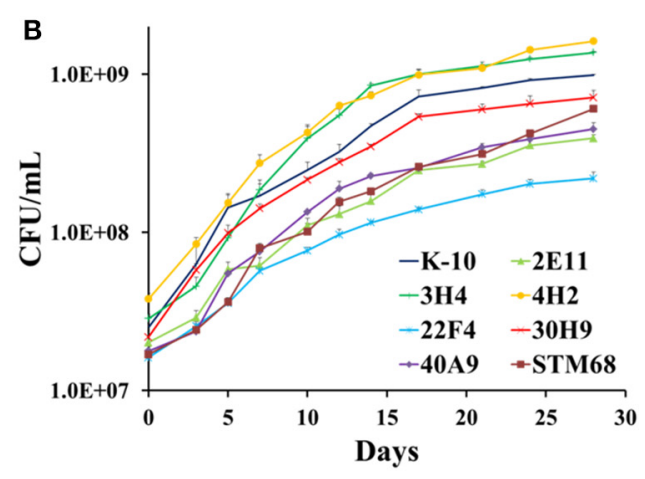

standing for 28 days. OD readings $(\mathbf{A})$ and CFU after 5 weeks $(\mathbf{B})$ were taken Results represent means $(n=3) \pm$ standard error of the mean. See text for relevant $P$-values. 


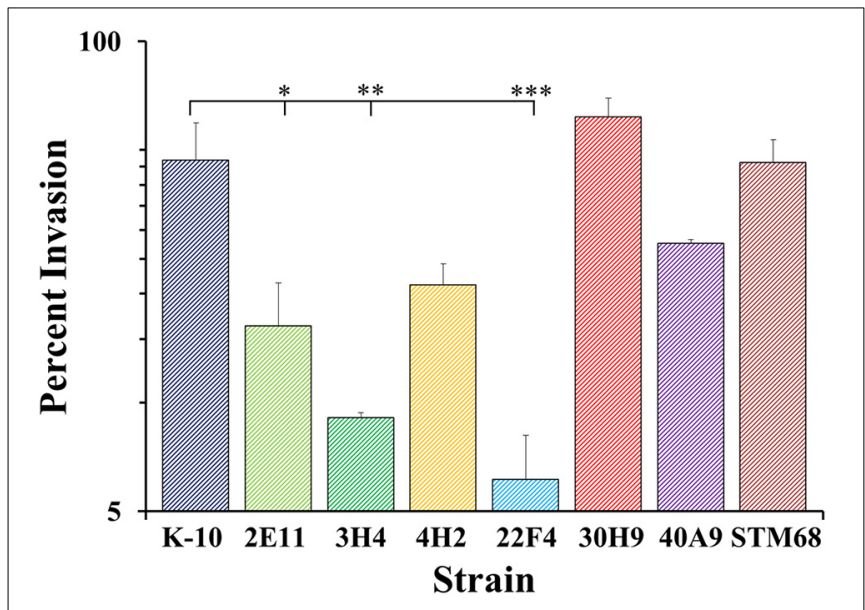

FIGURE 8 | Invasion of MAP wild type K-10 and mutant strains in bovine macrophages. Procedures followed are described in Lamont and Sreevatsan (2010). MAP uptake at the end of the invasion incubation period (percent invasion, $2 \mathrm{~h}$ after infection). Results represent means

$(n=3) \pm$ standard error of the mean. Statistical significance for pairwise determinations displayed by a line with tick marks is denoted with asterisks: ${ }^{*} P$-value $<0.05,{ }^{* *} P$-value $<0.01$ and ${ }^{* * *} P$-value $<0.001$.

in comparison to $\mathrm{K}-10 \mathrm{P}>0.1$. These results sharply contrast with strain invasiveness for BoMac cells (see Section Discussion).

The full short kinetics of the interaction of MAP strains with MDMs is shown in Figure 9. This analysis demonstrates that for most strains there is a pronounced killing phase immediately after invasion incubation up to $6-12 \mathrm{~h}$, followed by a recovery phase and eventual stabilization of the CFU burdens. This aspect of the interaction is usually missed in standard assays that only study 2 time points (immediately after invasion incubation and 2/4 days). In this context, the killing and intracellular replication rates may also influence the final outcome of the interaction of MAP and MDMs. Statistical analysis of each process (e.g., killing and replication-stabilization phases) depicted in these curves was performed. Results indicated that strains $4 \mathrm{H} 2(P<0.05)$ and 40A9 $(P<0.05)$ were killed much more rapidly than $\mathrm{K}-10$, while $22 \mathrm{~F} 4$ $(P<0.001)$ showed an unexpected increase in CFUs during the first $6 \mathrm{~h}$. The intracellular replication rates (evaluated between 12 and $48 \mathrm{~h}$ ) were similar for the wild type and all mutant strains, except for $3 \mathrm{H} 4(P<0.01)$ and $40 \mathrm{~A} 9(P<0.001)$ that grew faster than K-10.

Figure 10 displays CFU burdens at Day 0 after invasion incubation (striped bars) and Day 2 (solid bars) for mutant and wild type strains. As the MAP inoculums for MDMs were approximately the same for all strains, the CFU burdens at Day 0 are proportional to strain invasiveness (Figure 8). Thus, direct comparisons of Day $0 \mathrm{CFU}$ burdens yielded similar $P$ values for pair-wise comparisons as those observed for differences in invasion, except that the decrease in invasiveness for $3 \mathrm{H} 4(P<0.001)$ vs. K-10 had a greater significance level. Nonetheless, the analysis based on percent invasion presented in Figure $\mathbf{8}$ is more accurate, as any differences in the inoculums are taken into account. Regarding strain intracellular survival, as indicated by the CFU burdens for each strain at Day 0 and Day 2, no significant

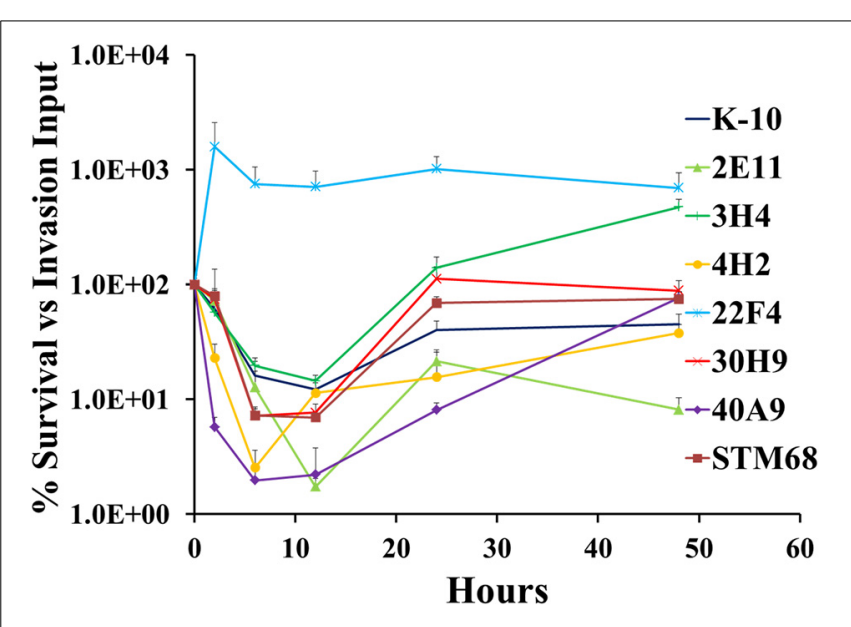

FIGURE 9 | Survival and replication of MAP wild type K-10 and mutant strains in bovine macrophages. Procedures followed are described in Lamont and Sreevatsan (2010). Kinetics of MAP intracellular survival and replication (after invasion) are based on the following formula:

$\%$ Survival $=\left(\mathrm{CFU} / \mathrm{ml}_{\text {time }, t} / \mathrm{CFU} / \mathrm{ml}_{\text {time }, \mathrm{t}=0}\right) \times 100 \%$; time, $t$ is any of the time points while time, $t=0$ is the time point immediately after completion of invasion incubation. Results represent means $(n=3) \pm$ standard error of the mean. See text for relevant $P$-values.

differences were observed for strains 30H9, 40A9, and STM68 $(P>0.1)$. For $\mathrm{K}-10$, the decrease observed in survival is moderate, but falls within statistical significance $(P<0.05)$. In contrast, marked decreases are observed for $2 \mathrm{E} 11(P<0.001)$ and $4 \mathrm{H} 2$ $(P<0.01)$. Interestingly, 3H4 $(P<0.001)$ and 22F4 $(P<0.001)$ were able to survive and replicate significantly better than $\mathrm{K}$ 10 intracellularly. Comparisons of the overall macrophage CFU burdens at Day 2 among all strains allow us to determine the combined effects of invasion and intracellular survival/replication. The results indicate that strain 2E11 is definitively attenuated in comparison to $\mathrm{K}-10(P<0.001)$; while $4 \mathrm{H} 2$ is slightly attenuated, but this difference is not statistically significant. Strains $22 \mathrm{~F} 4$, 40A9, and STM68 behaved similarly to K-10 $(P>0.1)$, while strains $3 \mathrm{H} 4(P<0.05)$ and $30 \mathrm{H} 9(P<0.05)$ seem to reach higher CFU burdens than $\mathrm{K}-10$.

In summary for the interaction of MAP strains with MDMs, mutants 2E11, 3H4, and 22F4 were less invasive than K-10. In relation to survival, $2 \mathrm{E} 11$ and $4 \mathrm{H} 2$ displayed marked reductions. For the overall Day 2 CFU burdens, only 2E11 was attenuated. Overall, strains 22F4, 40A9, and STM68 behaved similarly to K10 , while $3 \mathrm{H} 4$ and $30 \mathrm{H} 9$ reached a higher number of intracellular bacilli. For the killing phase, $4 \mathrm{H} 2$ and $40 \mathrm{~A} 9$ were killed faster than K-10 and all mutant strains were able to grow intracellularly at approximately the same rate, except for $3 \mathrm{H} 4$ and $40 \mathrm{~A} 9$ that replicated faster.

\section{Complementation analysis}

We also attempted to develop a complementation system for the Tn5367 transposon mutants using a derivative of the multi-copy plasmid pMV206_Hyg (George et al., 1995). To this end, vector pBUN277 was constructed carrying a hyg marker and the green fluorescent protein gene downstream from the mycobacterial heat shock promoter $h s p 60$. Assessment of wild type K-10, 


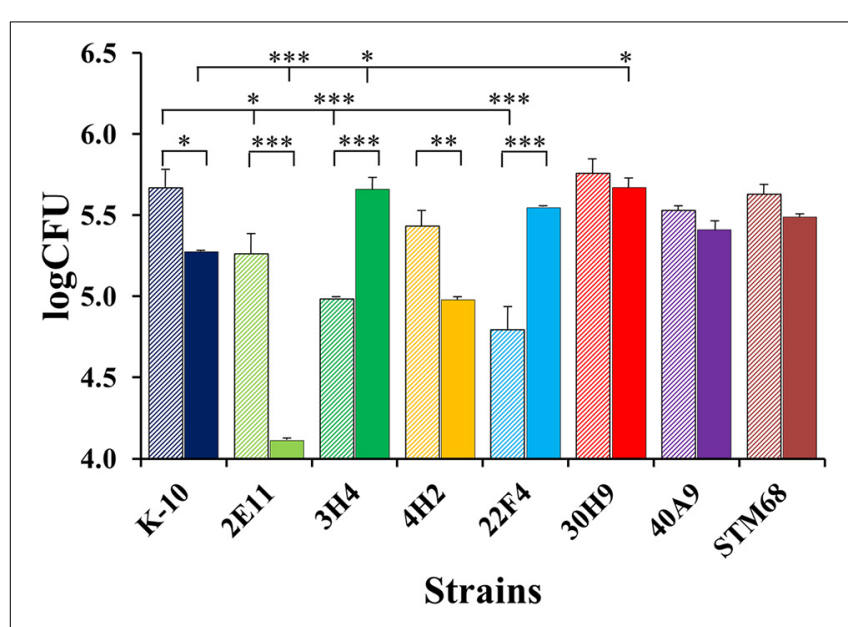

FIGURE 10 | MAP burdens of wild type K-10 and mutant strains in bovine macrophages. Procedures followed are described in Lamont and Sreevatsan (2010). Total CFU burdens at Day 0 ( $2 \mathrm{~h}$ after infection, striped bars) and Day 2 (solid bars) are displayed. Results represent means $(n=3) \pm$ standard error of the mean. Statistical significance for pairwise determinations displayed by a line with tick marks is denoted with asterisks: ${ }^{*} P$-value $<0.05,{ }^{* *} P$-value $<0.01$ and ${ }^{* * *} P$-value $<0.001$.

$4 \mathrm{H} 2$ and the empty vector control $4 \mathrm{H} 2$ (pBUN277) in MDMs yielded anomalous results. The survival of $4 \mathrm{H} 2$ was significantly different $(P<0.05)$ from $\mathrm{K}-10$ at 5 days post-infection, while the survival of $4 \mathrm{H} 2$ (pBUN277) was not significantly different $(P>0.05)$ from K-10 (unpublished results). In addition, the survival of $4 \mathrm{H} 2$ recombinant strains carrying either the wild type gene MAP1152 or MAP1156 yielded similar results to the empty vector control $(P>0.05)$. This data indicates that the complementation system developed was not appropriate and further attempts were no longer pursued for additional mutants.

\section{DISCUSSION}

The objective of our study was to generate a comprehensive MAP mutant bank and isolate strains with interesting phenotypic properties and identify genomic regions associated with virulence that could be targeted for further development of live-attenuated vaccine strains. Mutants identified for at least one phenotypic property potentially related to virulence are displayed in Table S1. This set of 123 strains represents ca. $1 \%$ of the mutant bank. The uniqueness of our approach was to perform the screen independently of homology searches for genes already identified in related mycobacterial species, so as to maximize hits in genes specific for MAP.

Statistical considerations, coupled with the demonstration that randomly picked mutants were independent, suggest that the library was comprehensive: e.g., mutants displayed unique hybridization bands to the IS1096 probe (see Section Southern hybridization) and diverse phenotypes (Table S1). It is especially noteworthy that five of the mutants selected for the vaccine trail $(3 \mathrm{H} 4,4 \mathrm{H} 2,22 \mathrm{~F} 4,30 \mathrm{H} 9$, and 40A9) and three additional mutants identified as attenuated in BoMac cells or preliminary MDM assays (12G8, 23B5, and 37D1) were all associated with altered DCS susceptibility. As for the rapid screens for cell association, and biofilm and clump formation, five (1B11, 3D9, 4E1, 4E7, and $4 \mathrm{~F} 3$ ) out of ca. 300 screened mutants (approximately 1.5\%) of the pool, identified strains with properties that may relate to virulence, but did not overlap with the DCS altered susceptibility set. Thus, implementation of additional screens, or expansion of the screens reported herein may still reveal a significant number of potentially attenuated mutants. However, the inherent bias of IS1096-derived transposons limits somewhat the comprehensiveness of the mutant bank (McAdam et al., 2002; Shin et al., 2006). Fortunately, Himar-derived transposons with more random recognition sequences have been developed for mycobacteria (Sassetti and Rubin, 2003) and are already in use for MAP (Scandurra et al., 2009; Wang et al., 2014). These vectors may provide a more representative mutant repertoire, but at the cost of a significant increase (ca. 10-fold) in the number of mutants that should be screened for a comprehensive analyses. In addition, best recommended screening approaches for Himar libraries are focused on gene identification analyses under specified selection pressures (Sassetti and Rubin, 2003). Individual deletion mutants and complementation analysis must then be performed to further confirm the role of these genes in virulence. Thus, the library described herein is useful as source of individual mutants for identification of genes involved in MAP pathogenesis, but implementation of additional screens to formally determine its comprehensiveness may not be warranted. Nonetheless, as described, we performed three comprehensive screens (colony morphology, DCS hypersusceptibility and siderophore production), and three additional limited screens for reduced biofilm and clump formation, and decreased cell association with BoMac cells. Assuming that most of these insertions are in different genes, this collection may represent insertions in ca. 120 genes. In this context, previous studies with MTB demonstrated that 194 genes are required for infection in mice (Sassetti and Rubin, 2003).

Seven Tn5367 mutants from the set depicted in Table S1 as well as STM68, a mutant from the Tn5370 signature-tagged mutagenesis pool, were further characterized and their properties are summarized in Table 2 . In our comprehensive screening of the mutant bank using a chromogenic assay for siderophore detection, only mutant 1F3 did not display a halo in the CAS plate assay. This mutant grew very slowly in broth cultures but was able to colonize goats (Livneh et al., 2005). Unfortunately, as indicated above, stocks in two laboratories failed to grow upon further attempts at replication and the transposon insertion site could not be determined. The CAS assay phenotype has been associated with impairment of siderophore synthesis or secretion in Msmeg (Fiss et al., 1994). Thus, the properties of 1F3 suggests that MAP has at least an additional siderophore-like molecule that may be involved in iron uptake and play important physiologic roles. Though MAP has a truncated $m b t A$ gene that most likely underlies its inability to synthesize mycobactin (Li et al., 2005), mycobactin-dependence is observed mainly in MAP cultured directly from primary lesions or other clinical specimens. In passaged cultures, mycobactin dependence can be readily overcome in acidic iron-rich media (Lambrecht and Collins, 1992). Thus, we hypothesized that 1F3 may have had a lesion in a gene encoding for a siderophore-like molecule other than mycobactin. 
Table 2 | Screening and characterization of MAP strains.

\begin{tabular}{|c|c|c|c|}
\hline Strain $^{a}$ & ORF(s)-Mutated or Affected ${ }^{b}$ & ORFs Function ${ }^{c}$ & Original Screen and Further Comments ${ }^{d}$ \\
\hline 1F3 & Tn5367 insertion was not identified & Not applicable & $\begin{array}{l}\text { Chromogenic screen for siderophore synthesis or } \\
\text { transport; mutant lost upon subsequent passages }\end{array}$ \\
\hline $\begin{array}{l}2 \mathrm{E} 11 \\
\text { Vaccine } \\
316\end{array}$ & $\begin{array}{l}\text { Tn5367 between } \\
\text { MAP3695 (Rv0245) and FadE5 } \\
\text { (Rv0244c) }\end{array}$ & $\begin{array}{l}\text { MAP3695 (hypothetical protein) } \\
\text { FadE5 (terpenoid and polyketide metabolism) }\end{array}$ & $\begin{array}{l}\text { Attenuated in MDMs for invasion and intracellular } \\
\text { survival }\end{array}$ \\
\hline $\begin{array}{l}4 \mathrm{H} 2 \\
\text { Vaccine } \\
321\end{array}$ & $\begin{array}{l}\text { Tn5367 between MAP1150c } \\
\text { (Rv3640c) and MAP1151c (Rv2839c) } \\
\text { Upstream from MAP1152 } \\
\text { (Rv1808)-MAP1156 (Rv1425) cluster }\end{array}$ & $\begin{array}{l}\text { MAP1150c (IS } 1311 \text { transposase) } \\
\text { MAP1151c (translational initiation factor IF2) } \\
\text { MAP1152 (PPE protein) } \\
\text { MAP1156 (diacyglycerol O-acyltransferase) }\end{array}$ & $\begin{array}{l}\text { Colony-morphology mutant, attenuated for invasion } \\
\text { in BoMac cells, and invasion and survival in MDMs. } \\
\text { Mutant also displayed DCS hypersusceptibility. }\end{array}$ \\
\hline $\begin{array}{l}30 \mathrm{H} 9 \\
\text { Vaccine } \\
319\end{array}$ & $\begin{array}{l}\text { Tn5367 within } \\
\text { MAP1566 (Rv1645c) near 5'-end } \\
\text { coding sequence }\end{array}$ & $\begin{array}{l}\text { MAP1566 } \\
\text { ORF downstream from ModA (MAP1565) in } \\
\text { the mod operon }\end{array}$ & $\begin{array}{l}\text { DCS hypersusceptible; attenuated for invasion in } \\
\text { BoMac cells }\end{array}$ \\
\hline $\begin{array}{l}40 A 9 \\
\text { Vaccine } \\
318\end{array}$ & $\begin{array}{l}\text { Tn5367 between MAP0282c } \\
\text { (Rv3860) and MAP0283c (Rv2082) }\end{array}$ & $\begin{array}{l}\text { MAP0282c and MAP0283c (hypothetical } \\
\text { proteins, assigned with low confidence); or } \\
\text { spontaneous rRNA mutations }\end{array}$ & $\begin{array}{l}\text { Decreased susceptibility to DCS; transposon lost } \\
\text { upon further passage; attenuated in MDMs by } \\
\text { increased killing rate }\end{array}$ \\
\hline $\begin{array}{l}\text { STM68 } \\
\text { Vaccine } \\
315\end{array}$ & $\begin{array}{l}\text { Tn5370 within } \\
\text { MAP1566 (Rv1645c) near 3'-end } \\
\text { coding sequence }\end{array}$ & $\begin{array}{l}\text { MAP1566 } \\
\text { ORF downstream from ModA (MAP1565) in } \\
\text { the mod operon }\end{array}$ & $\begin{array}{l}\text { Identified as MAP1566 insertion by direct } \\
\text { sequencing of Tn5370 signature-tagged } \\
\text { mutagenesis pool }\end{array}$ \\
\hline
\end{tabular}

a Laboratory designation followed by the vaccine trial designation (Bannantine et al., 2014; Hines et al., 2014).

${ }^{b}$ MAP ORF designation is followed by MTB definite or putative ortholog in parenthesis.

c See text for citations on ORFs functions as applicable.

${ }^{d}$ See text for further details.

Mutant 2E11 was a false positive for reduced biofilm formation, but it was nonetheless selected for further studies, since preliminary assays indicated attenuation in MDMs. Assignment of the target gene for mutant $2 \mathrm{E} 11$ was not fully resolved in this study as the Tn5367 insertion was mapped to the intergenic region (Figure 5) encoding an acyl-coA dehydrogenase (MAP3694c, aka FadE5) and a possible oxidoreductase (MAP3695). Interestingly, FadE5 is involved in lipid degradation (http://www.ncbi.nlm.nih.gov/biosystems/1743) including the metabolism of terpenoids and polyketides, such as geraniol, and these compound types have been associated with biofilm formation in bacteria (Zheng et al., 2013). MAP FadE5 is orthologous to MTB Rv0244c, which has been shown to be required for in vitro growth on cholesterol (http://tuberculist.epfl.ch). This mutant was the most attenuated in this study on account of both invasiveness and survival in MDMs.

Mutant 3H4 displayed a transposon insertion in the intergenic region between MAP2296c and MAP2297c. Both these ORFs encode for hypothetical proteins, with the MTB ortholog (Rv2478c) possibly encoding a non-essential DNA binding protein, and MAP2297c ortholog (Rv0102) identified as an integral essential membrane protein and $\mathrm{ABC}$ transporter that may underlie the DCS hypersusceptible phenotype (http://tuberculist. epfl.ch). Though, this mutant was originally selected as a normal colony morphotype control, it displayed hypersusceptibility to DCS. Nonetheless, this strain behaved similarly to the wild type K-10 strain in both BoMac cells and MDMs, except that it displayed an attenuated invasion phenotype in the interaction with MDMs that was compensated with a higher intracellular replication rate.

In $4 \mathrm{H} 2$, the transposon inserted within the intergenic region between MAP1150c and MAP1151c. MAP1150c is the transposase for IS1311 (Murcia et al., 2007), an insertion sequence present at eight copies in the MAP genome (Li et al., 2005). Thus, any effect of this insertion on the MAP1150c is not likely to have a major impact on MAP physiology. In addition, the insertion may not have an effect on MAP1151c either as the transposon is located beyond the $3^{\prime}$-end of this coding sequence. However, there is a gene cluster (MAP1152-MAP1156) (Bannantine et al., 2011) that is transcribed from the complementary strand to MAP1150c, 
whose expression could be effected by the transposon insertion that is located $634 \mathrm{bp}$ upstream from the $5^{\prime}$-end of MAP1152. This gene cluster is very interesting since MAP1152, MAP1153 and MAP 1155 encode PPE proteins of the ancestral sublineage IV (Gey Van Pittius et al., 2006). PPE proteins have been suggested to play a role as host range determinants (Cole et al., 1998) and the immunopathogenesis of both humoral and cellular responses in MTB (Choudhary et al., 2003; Okkels et al., 2003) and MAP (Nagata et al., 2005). Other PPE proteins in MTB (Rv1807, Rv3872, and Rv3873) have been implicated in virulence since inactivation of the corresponding genes results in mutants attenuated in mice (Sassetti and Rubin, 2003). In addition, PPE and $\mathrm{PE}$ proteins function in pairwise combinations of interacting partners exposed to the cell surface (Riley et al., 2008) and both sets are markedly underrepresented in MAP (cf. 36 PPE proteins in MAP vs. 68 in MTB) (Li et al., 2005). The other members of the gene cluster, MAP1154 and MAP1156, encode proteins of an uncharacterized protein family. MAP1154 encodes a hypothetical protein, while MAP1156 has putative diacyglycerol O-acyltransferase activity (Bannantine et al., 2011). Mutant 4H2 was clearly attenuated in both BoMac and MDM cells, on account of reduced invasiveness and intracellular survival. Moreover, we have demonstrated that MAP1152 and MAP1156 engender humoral immunity responses that can be used to identify infected animals and thus, the corresponding mutants have potential for DIVA (differentiating infected from vaccinated animals) vaccine formulations (Bannantine et al., 2011).

Mutants 22F4 and 30H9 were identified as DCS hypersusceptible strains. Isolate 22F4 carries an insertion within the gene encoding the Lsr2 protein that has been implicated in various roles: dominant $\mathrm{T}$ cell responses in MTB (Stewart et al., 2002) and biofilm formation and colony morphology in Msmeg (Chen et al., 2006). Furthermore, a MAP lsr2 deletion mutant has been constructed (Park et al., 2008) that showed similar properties and a marginal degree of attenuation in MDMs to 22F4 (Lamont et al., 2014), thus providing strong evidence that the transposon insertion, that is internal to $l s r 2$, does disrupt the function of this gene.

Strain $30 \mathrm{H} 9$ has an insertion internal to MAP1566 and proximal to its $5^{\prime}$-end coding sequence. Likewise, STM68 carries an insertion in MAP1566 but closer to its $3^{\prime}$-end that could potentially have a greater effect on the downstream gene MAP1567. The cluster MAP1565-MAP1568 seems to comprise an operon encoding a high affinity molybdate transport system, as indicated by bioinformatic analysis (Braibant et al., 2000). Orthologs are present in M. avium strain 104 (MAV_2862, 99\% identity), MTB (Rv1645c, 35\% identity, 50\% similarity) as well as other mycobacterial species. However, only the MAP and M. avium 104 strain orthologs are located within the Mod operon, making this arrangement unique to these species. We hypothesize that MAP1566 could be a chaperone that works in concert with this transport system, while MAP1565 (ModA) and MAP1567 (ModB) are integral membrane proteins that constitute and stabilize the transport channel. It seems that MAP1569 (ModD) has a function in fibronectin binding unrelated to this molybdenum transport system (Kumar et al., 2003) and thus it may correspond to a different transcriptional unit. Strong evidence for the transposon insertions affecting the functions of Mod operon (e.g., MAP1566 or MAP1567) is provided by the similar properties of both mutants in MDMs, mice (Bannantine et al., 2014) and goats (Hines et al., 2014). These mutants were attenuated in C57BL/6 mice but protective against wild type challenge, displayed low skin reactivity in goats and showed significant attenuation in MDM assays as analyzed elsewhere (Lamont et al., 2014). However, in this current analysis, 30H9 and STM68 were comparable to the wild type strain. Interestingly, another MAP1566 Tn5367 mutant (WAg906), derived from the MAP wild type strain 989 (Cavaignac et al., 2000), was shown to be attenuated based on the average slope values obtained in long-term MDM assays as well as $\mathrm{BALB} / \mathrm{c}$ mice at 12 weeks post-infection (Scandurra et al., 2010; Bannantine et al., 2014; Lamont et al., 2014). However, in protection challenge experiments, 30H9 and STM68 showed good protection in $\mathrm{C} 57 \mathrm{BL} / 6$ mice against wild type challenge, while WAg906 failed to protect BALB/c mice. Use of different MAP and mouse strains may explain these results. These three mutants were attenuated for survival in goats, but only $30 \mathrm{H} 9$ and STM68 were tested for protection against challenge, displaying low efficacy (Scandurra et al., 2010; Hines et al., 2014).

As for 40A9, the loss of the transposon insertion does not allow us to make any definitive conclusions on the molecular basis underlying its attenuation. However, this mutant still retained the Kan-resistant phenotype suggesting the involvement of spontaneous mutations in ribosomal RNA genes (Du et al., 2013). Nonetheless, this mutant was the most protective from this collection in the goat trial (Hines et al., 2014). The most interesting property observed for this strain was a fast intracellular killing in MDMs followed by its ability to recover and grow (Figure 9), but otherwise this strain reached a similar CFU burden as K-10 at Day 2 post-infection (Figure 10).

Regarding the complementation studies, there are several reasons that may explain the anomalous results including: (i) the hyg marker may increase the survival of the host strains as it was observed for Msmeg recombinant strains (Chacon et al., 2009); (ii) the inherent instability of the Tn5367 mutant strains with the potential for further transposition events by a cut and paste mechanism (McAdam et al., 1995); (iii) the possibility of spurious recombination events for strains carrying multiple copies of wild type DNA sequences in the complementing plasmids and the transposon interrupted copies in the genome. Due to these difficulties, conclusions regarding the roles of the genes identified in this study are still presumptive rather than definitive. Nonetheless, the bioinformatic analysis and the correlation with our previous results and those from other laboratories increase the confidence that most of these genes are relevant to MAP survival and pathogenesis. Moreover, MAP slow growth and low transformation efficiency place an onerous time demand on further attempts to revisit complementation studies (Foley-Thomas et al., 1995). These issues may underlie the lack of reported complementation analysis in previous MAP transposon mutant reports (Shin et al., 2006; Scandurra et al., 2009, 2010). To overcome these complications, we are currently implementing more solid strategies to construct and complement deletion mutants using different selection markers. 
An important objective of this study was to compare the interaction of different MAP strains with BoMac cells and MDMs in this study and the parallel study in the vaccine trial (Lamont et al., 2014). In our studies, the order of attenuation in BoMac cells and MDM cultures (comparing invasion percentiles and slopes for each infection phase) was significantly different. Strain $30 \mathrm{H} 9$ displayed the greatest attenuation in BoMac cells, while it was the least attenuated in the MDM assays based on the analysis reported herein. Mutants $3 \mathrm{H} 4$ and 22F4 yielded less discrepancy, while $4 \mathrm{H} 2$ behaved similarly. However, when the strains were compared only by the average slope values in MDM assays (Lamont et al., 2014), results were closer to those obtained with BoMac cells, with $30 \mathrm{H} 9$ yielding the greatest attenuation among the four strains that were also tested in BoMac cells. Notably, 3H4, 22F4, and $30 \mathrm{H} 9$ had major differences in invasiveness for the two cell types: $3 \mathrm{H} 4$ and 22F4 were invasive for BoMac cells, but not for MDMs (Figures 3, 8), while the opposite was observed for $30 \mathrm{H} 9$. The differences observed are likely due to the inherent nature of the BoMac cell line that has poor phagocytosis and decreased ability to sustain intracellular MAP replication, as shown previously for the wild type strain K-10 (Woo et al., 2006). A similar situation seems to occur in MDM aging cultures (Scandurra et al., 2010; Lamont et al., 2014). Thus, determination of the early kinetics at each phase (e.g., invasion, intracellular killing and replication), as done in this study, seems to provide the best assessment of strain attenuation for studies in ruminants. This analysis may be supplemented with assays to determine apoptosis and measure cytokines such as IL-10 (Scandurra et al., 2010; Kabara and Coussens, 2012; Lamont et al., 2014).

Comparing the results from this study with the recent vaccine trial, though not conclusive, sheds some light on the relation among the various models and vaccine protection for ruminants. Attenuation in BoMac cells and analysis of average slopes in MDM assays showed significant correlation with attenuation and protection in $\mathrm{C} 57 \mathrm{BL} / 6$ mice vaccinated by intraperitoneal injection, but not with orally vaccinated goats (Bannantine et al., 2014; Hines et al., 2014; Lamont et al., 2014). In contrast, the analysis in this manuscript of MDM assays correlated well with protection results in goats. Indeed, the four strains tested in goats ranked similarly; except for the inversion between 2E11 (most attenuated in MDMs as reported here, but ranked 2nd in goats) and 40A9 (ranked 2nd in MDMs, but most protective in goats).

As to the MAP mutant properties necessary to elicit protective immunity in ruminants, based on the conditions tested in the vaccine trial, interplay between attenuation and virulence seems necessary. Moreover, a level of persistence (e.g., able to colonize well in vivo at early time points but lead to significant reduction in colonization at later times post-infection) has been hypothesized as one desirable property for the design of MTB live attenuated vaccines (Hingley-Wilson et al., 2003). For example, 40A9 would be considered rather wild type like, based on the CFU burdens at either Day 0 or Day 2 (Figure 10). However, this strain displays both faster killing and intracellular replication rates (Figure 9). Significant killing of 40A9 in their first encounter with macrophages may provide sufficient attenuation while preserving protective immunogenicity. The subsequent fast intracellular recovery may lead to a persistent behavior. Though,
40A9 was not tested in BALB/c mice for the persistent phenotype, this behavior was observed for Vaccine 329 that outperformed 40A9 in goats (Shin et al., 2006; Hines et al., 2014). In contrast, $30 \mathrm{H} 9$ and STM68 displayed a behavior similar to the wild type K-10 in each of the steps involved in the interaction of the mutant strains with MDMs (Figures 8-10), but performed worse in the goat vaccination trial. In addition, little correlation was observed between the mouse and goat trails, though in this case the more susceptible C57BL/6 mice were used (Bannantine et al., 2014). Thus, the level of protection of a candidate vaccine strain may depend on earlier interactions of the mutant strains with the oral, gastric and intestinal mucosa, and intracellular killing and replication rates in MDMs (Bermudez et al., 2010). In addition, there still remains significant issues regarding tissue culture (BoMac vs. MDM cells), analysis of tissue culture results, animal model (BALB/c and C57BL/6 mice or goats), dose (low vs. high) and routes of inoculation (intraperitoneal, oral and subcutaneous) that need further testing and standardization to make better predictions on protection for JD vaccines in cattle.

\section{CONCLUSIONS}

The major accomplishments and findings of this study are: (1) a comprehensive MAP Tn5367 transposon mutant bank was constructed by indexing a large collection of independent mutants that was statistically representative of the full genome; (2) Tn5367 mutants were inherently unstable due to the IS1096 transposase presence within the transposed sequence; (3) phenotypic screens were effective in identifying attenuated mutants or processes of importance for pathogenesis or cell physiology: (a) the presence of additional siderophores other than mycobactin (e.g., 1F3), (b) the association of colony morphotypes with reduced virulence (e.g., $4 \mathrm{H} 2$ and 22F4), (c) the association of drug-susceptible (e.g., 22F4 and 30H9) or resistant (e.g., 4H2 and 40A9) phenotypes with reduced virulence and (d) the potential role of cell association, and biofilm and clump formation with virulence (see Section Miscellaneous screening); (4) mutant screening in BoMac cells and MDMs yields significantly different results; (5) strain invasiveness and slope values at each step in the interaction of MAP bacilli with MDMs, especially the slopes during the early killing phase, are the best predictors for protection against wild type challenge in the oral goat vaccination model; (6) the interaction of MAP with MDMs is complex and key aspects of this process involve: invasion, intracellular killing, intracellular replication and maintenance of CFU intracellular burdens; (7) mutant screening with BoMac cells may have significant biases and it should be abandoned as a routine methodology to assess attenuation; and (8) the most important stages of MAP interaction with MDMs in tissue culture occur at relatively short times (up to $48 \mathrm{~h}$ post-invasion incubation). Our results suggests that further studies with MAP mutants should focus on early step-wise analysis of their interactions with MDMs and the use of transposon delivery vectors that lack the transposase within the transposed element (e.g., Tn5370 and the newly developed Himar1 derivatives).

\section{ACKNOWLEDGMENTS}

The following Federal Agency sources are acknowledged for funding: USDA CSREES-NRI (99-35204-7789, 2004-35204-14231 and 
JDIP 2004-35605-14243), USDA-BARD (IS-3673-05C), USDANIFA Animal Health ARD Station Project (NEB-14-141 and NEB-39-162). We also wish to acknowledge worthwhile discussions and advice from Drs. Luiz Bermudez (MDM assays), Ofelia Chacon (DCS screen), Jeffrey D. Cirillo (Tn5370 phage materials and sequencing data), Amit Pandey (complementing plasmid constructions), Dilip Patel (MDM blind assays for complementation studies in Bermudez laboratory) and William R. Jacobs Jr. (various tools for mycobacterial genetic manipulations). We thank Shingo Ishihara and Harshdeep Dogra for technical assistance in preliminary PCR experiments and vector construction, respectively. Strains described in Table 2 of this manuscript are available from the corresponding author's laboratory or the laboratories of the USDA-CAP Johne's Integrated Program (JDIP) sponsored vaccine trial participating institutions (Bannantine et al., 2014; Hines et al., 2014; Lamont et al., 2014) upon signing a modified Uniform Biological Material Transfer Agreement via Pennsylvania State University.

\section{SUPPLEMENTARY MATERIAL}

The Supplementary Material for this article can be found online at: http://www.frontiersin.org/journal/10.3389/fcimb. 2014.00144/abstract

Figure S1 | (A-D) Screening of MAP mutants for susceptibility or resistance to DCS. All mutants were plated on MOADC-Plus media without $(\mathbf{A}, \mathbf{C})$ and with $(\mathbf{B}, \mathbf{D})$ DCS at $20 \mu \mathrm{g} / \mathrm{ml}$ and allowed to grow for 8 weeks. There are two different scale bars displayed since a Fisherbrand 08-757-14 round (A,C) or Corning 431110 square (B,D) plate was utilized. Mutants with altered DCS susceptibility are circled with black outlines: the rough colony morphotype $4 \mathrm{H} 2(\mathbf{A}, \mathbf{B})$ displays a resistant phenotype while the smooth colony morphotype 22F4 (C,D) displays hypersusceptibility.

Figure S2 | Rapid screening for MAP mutants with decreased biofilm formation. The left image depicts a MAP mutant with reduced biofilm formation while the image on the right is a mutant with normal biofilm formation.

Figure S3 | (A,B) Rapid screening for MAP mutants with reduced cell association. A rapid in vitro assay was developed to screen the library for mutants with decreased cell association (e.g., less adherence and/or invasion) with BoMac cells. Cells were plated on a 16-well chamber slide and infected with MAP wild type and mutant strains. At $24 \mathrm{~h}$ post-incubation, slides were fixed and acid-fast bacteria were stained by the Auramine-Rhodamine method that yields green fluorescence for acid-fast bacilli. Representative microscopic images from an infection with the wild type strain K-10 (A) and a mutant with decreased cell association (B) are shown. Internal scale bar is $100 \mu \mathrm{m}$.

Figure S4 | (A,B) Rapid screening for MAP mutants with reduced clump formation. To test for mutants with reduced clump formation, a property that could be related to virulence, broth cultures were analyzed by microscopy. Microscopic images of the wild type strain K-10 (A) and a mutant with reduced clump formation (B) are shown. Internal scale bar is $10 \mu \mathrm{m}$.

\section{REFERENCES}

Bach, H., Sun, J., Hmama, Z., and Av-Gay, Y. (2006). Mycobacterium avium subsp. paratuberculosis PtpA is an endogenous tyrosine phosphatase secreted during infection. Infect. Immun. 74, 6540-6546. doi: 10.1128/IAI.01106-06

Bannantine, J. P., Everman, J., Rose, S. J., Babrak, L., Katani, R., Barletta, R. G., et al. (2014). Evaluation of eight live attenuated Mycobacterium avium subspecies paratuberculosis mutant strains for protection from colonization of tissues in mice. Front. Cell. Infect. Microbiol. 4:88. doi: 10.3389/fcimb.2014.00088

Bannantine, J. P., Paulson, A. L., Chacon, O., Fenton, R. J., Zinniel, D. K., Mcvey, D. S., et al. (2011). Immunogenicity and reactivity of novel Mycobacterium avium subsp. paratuberculosis PPE MAP1152 and conserved MAP1156 proteins with sera from experimentally and naturally infected animals. Clin. Vaccine Immunol. 18, 105-112. doi: 10.1128/CVI.00297-10

Bannantine, J. P., and Paustian, M. L. (2006). Identification of diagnostic proteins in Mycobacterium avium subspecies paratuberculosis by a whole genome analysis approach. Methods Mol. Biol. 345, 185-196. doi: 10.1385/1-59745-143-6:185

Bannantine, J. P., Wu, C. W., Hsu, C., Zhou, S., Schwartz, D. C., Bayles, D. O., et al. (2012). Genome sequencing of ovine isolates of Mycobacterium avium subspecies paratuberculosis offers insights into host association. BMC Genomics 13:89. doi: 10.1186/1471-2164-13-89

Bardarov, S., Bardarov, S. Jr., Pavelka, M. S. Jr., Sambandamurthy, V., Larsen, M., Tufariello, J., et al. (2002). Specialized transduction: an efficient method for generating marked and unmarked targeted gene disruptions in Mycobacterium tuberculosis, M. bovis BCG and M. smegmatis. Microbiology 148, 3007-3017.

Bardarov, S., Kriakov, J., Carriere, C., Yu, S., Vaamonde, C., McAdam, R. A., et al. (1997). Conditionally replicating mycobacteriophages: a system for transposon delivery to Mycobacterium tuberculosis. Proc. Natl. Acad. Sci. U.S.A. 94, 10961-10966. doi: 10.1073/pnas.94.20.10961

Bastida, F., and Juste, R. A. (2011). Paratuberculosis control: a review with a focus on vaccination. J. Immune Based Ther. Vaccines 9:8. doi: 10.1186/1476-8518-9-8

Bermudez, L. E., Petrofsky, M., Sommer, S., and Barletta, R. G. (2010). Peyer's patch-deficient mice demonstrate that Mycobacterium avium subsp. paratuberculosis translocates across the mucosal barrier via both $\mathrm{M}$ cells and enterocytes but has inefficient dissemination. Infect. Immun. 78, 3570-3577. doi: 10.1128/IAI.01411-09

Braibant, M., Gilot, P., and Content, J. (2000). The ATP binding cassette (ABC) transport systems of Mycobacterium tuberculosis. FEMS Microbiol. Rev. 24, 449-467. doi: 10.1111/j.1574-6976.2000.tb00550.x

Bull, T. J., Schock, A., Sharp, J. M., Greene, M., Mckendrick, I. J., Sales, J., et al. (2013). Genomic variations associated with attenuation in Mycobacterium avium subsp. paratuberculosis vaccine strains. BMC Microbiol. 13:11. doi: 10.1186/1471-2180-13-11

Cavaignac, S. M., White, S. J., De Lisle, G. W., and Collins, D. M. (2000). Construction and screening of Mycobacterium paratuberculosis insertional mutant libraries. Arch. Microbiol. 173, 229-231. doi: 10.1007/s002039900132

Chacon, O., Bermudez, L. E., Zinniel, D. K., Chahal, H. K., Fenton, R. J., Feng, Z., et al. (2009). Impairment of D-alanine biosynthesis in Mycobacterium smegmatis determines decreased intracellular survival in human macrophages. Microbiology 155, 1440-1450. doi: 10.1099/mic.0.024901-0

Chacon, O., Feng, Z., Harris, N. B., Caceres, N. E., Adams, L. G., and Barletta, R. G. (2002). Mycobacterium smegmatis D-Alanine Racemase Mutants Are Not Dependent on D-Alanine for Growth. Antimicrob. Agents Chemother. 46, 47-54. doi: 10.1128/AAC.46.2.47-54.2002

Chen, J. M., German, G. J., Alexander, D. C., Ren, H., Tan, T., and Liu, J. (2006). Roles of Lsr2 in colony morphology and biofilm formation of Mycobacterium smegmatis. J. Bacteriol. 188, 633-641. doi: 10.1128/JB.188.2.633-641.2006

Chen, J. W., Faisal, S. M., Chandra, S., Mcdonough, S. P., Moreira, M. A., Scaria, J., et al. (2012a). Immunogenicity and protective efficacy of the Mycobacterium avium subsp. paratuberculosis attenuated mutants against challenge in a mouse model. Vaccine 30, 3015-3025. doi: 10.1016/j.vaccine.2011.11.029

Chen, J. W., Scaria, J., and Chang, Y. F. (2012b). Phenotypic and transcriptomic response of auxotrophic Mycobacterium avium subsp. paratuberculosis leuD mutant under environmental stress. PLoS ONE 7:e37884. doi: 10.1371/journal.pone.0037884

Cho, D., Shin, S. J., Talaat, A. M., and Collins, M. T. (2007). Cloning, expression, purification and serodiagnostic evaluation of fourteen Mycobacterium paratuberculosis proteins. Protein Expr. Purif. 53, 411-420. doi: 10.1016/j.pep.2006.12.022

Choudhary, R. K., Mukhopadhyay, S., Chakhaiyar, P., Sharma, N., Murthy, K. J., Katoch, V. M., et al. (2003). PPE antigen Rv2430c of Mycobacterium tuberculosis induces a strong B-cell response. Infect. Immun. 71, 6338-6343. doi: 10.1128/IAI.71.11.6338-6343.2003

Cirillo, J. D., Barletta, R. G., Bloom, B. R., and Jacobs, W. R. Jr. (1991). A novel transposon trap for mycobacteria: isolation and characterization of IS1096. J. Bacteriol. 173, 7772-7780. 
Cole, S. T., Brosch, R., Parkhill, J., Garnier, T., Churcher, C., Harris, D., et al. (1998). Deciphering the biology of Mycobacterium tuberculosis from the complete genome sequence. Nature 393, 537-544. doi: 10.1038/31159

Connell, N. D., Medina-Acosta, E., Mcmaster, W. R., Bloom, B. R., and Russell, D. G. (1993). Effective immunization against cutaneous leishmaniasis with recombinant bacille Calmette-Guerin expressing the Leishmania surface proteinase gp63. Proc. Natl. Acad. Sci. U.S.A. 90, 11473-11477. doi: 10.1073/pnas.90.24.11473

Cox, J. S., Chen, B., Mcneil, M., and Jacobs, W. R. Jr. (1999). Complex lipid determines tissue-specific replication of Mycobacterium tuberculosis in mice. Nature 402, 79-83.

Du, Q., Dai, G., Long, Q., Yu, X., Dong, L., Huang, H., et al. (2013). Mycobacterium tuberculosis rrs A1401G mutation correlates with high-level resistance to kanamycin, amikacin, and capreomycin in clinical isolates from mainland China. Diagn. Microbiol. Infect. Dis. 77, 138-142. doi: 10.1016/j.diagmicrobio.2013.06.031

Faisal, S. M., Chen, J. W., Yan, F., Chen, T. T., Useh, N. M., Yan, W., et al. (2013). Evaluation of a Mycobacterium avium subsp. paratuberculosis leuD mutant as a vaccine candidate against challenge in a caprine model. Clin. Vaccine Immunol. 20, 572-581. doi: 10.1128/CVI.00653-12

Feng, Z., and Barletta, R. G. (2003). Roles of Mycobacterium smegmatis D-alanine: $\mathrm{D}$-alanine ligase and $\mathrm{D}$-alanine racemase in the mechanisms of action of and resistance to the peptidoglycan inhibitor D-cycloserine. Antimicrob. Agents Chemother. 47, 283-291. doi: 10.1128/AAC.47.1.283-291.2003

Feng, Z., Caceres, N. E., Sarath, G., and Barletta, R. G. (2002). Mycobacterium smegmatis L-alanine dehydrogenase (Ald) is required for proficient utilization of alanine as a sole nitrogen source and sustained anaerobic growth. J. Bacteriol. 184, 5001-5010. doi: 10.1128/JB.184.18.5001-5010.2002

Fiss, E. H., Yu, S., and Jacobs, W. R. Jr. (1994). Identification of genes involved in the sequestration of iron in mycobacteria: the ferric exochelin biosynthetic and uptake pathways. Mol. Microbiol. 14, 557-569. doi: 10.1111/j.13652958.1994.tb02189.x

Foley-Thomas, E. M., Whipple, D. L., Bermudez, L. E., and Barletta, R. G. (1995). Phage infection, transfection and transformation of Mycobacterium avium complex and Mycobacterium paratuberculosis. Microbiology 141(Pt. 5), 1173-1181. doi: 10.1099/13500872-141-5-1173

George, K. M., Yuan, Y., Sherman, D. R., and Barry, C. E. 3rd. (1995). The biosynthesis of cyclopropanated mycolic acids in Mycobacterium tuberculosis. Identification and functional analysis of CMAS-2. J. Biol. Chem. 270, 27292-27298. doi: 10.1074/jbc.270.45.27292

Gey Van Pittius, N. C., Sampson, S. L., Lee, H., Kim, Y., Van Helden, P. D., and Warren, R. M. (2006). Evolution and expansion of the Mycobacterium tuberculosis PE and PPE multigene families and their association with the duplication of the ESAT-6 (esx) gene cluster regions. BMC Evol. Biol. 6:95. doi: 10.1186/1471-2148-6-95

Halouska, S., Fenton, R. J., Zinniel, D. K., Marshall, D. D., Barletta, R. G., and Powers, R. (2014). Metabolomics analysis identifies d-Alanine-d-Alanine ligase as the primary lethal target of d-Cycloserine in mycobacteria. J. Proteome Res. 13, 1065-1076. doi: 10.1021/pr4010579

Harris, N. B., Feng, Z., Liu, X., Cirillo, S. L., Cirillo, J. D., and Barletta, R. G. (1999). Development of a transposon mutagenesis system for Mycobacterium avium subsp. paratuberculosis. FEMS Microbiol. Lett. 175, 21-26. doi: 10.1111/j.15746968.1999.tb13597.x

Hines, M. E. 2nd., Turnquist, S. E., Ilha, M. R., Rajeev, S., Jones, A. L., Whittington, L., et al. (2014). Evaluation of novel oral vaccine candidates and validation of a caprine model of Johne's Disease. Front. Cell. Infect. Microbiol. 4:26. doi: 10.3389/fcimb. 2014.00026

Hingley-Wilson, S. M., Sambandamurthy, V. K., and Jacobs, W. R. Jr. (2003). Survival perspectives from the world's most successful pathogen, Mycobacterium tuberculosis. Nat. Immunol. 4, 949-955. doi: 10.1038/ ni981

Kabara, E., and Coussens, P. M. (2012). Infection of Primary Bovine Macrophages with Mycobacterium avium Subspecies paratuberculosis Suppresses Host Cell Apoptosis. Front. Microbiol. 3:215. doi: 10.3389/fmicb.2012.00215

Kumar, P., Amara, R. R., Challu, V. K., Chadda, V. K., and Satchidanandam, V. (2003). The Apa protein of Mycobacterium tuberculosis stimulates gamma interferon-secreting CD4+ and CD8+ T cells from purified protein derivativepositive individuals and affords protection in a guinea pig model. Infect. Immun. 71, 1929-1937. doi: 10.1128/IAI.71.4.1929-1937.2003
Lambrecht, R. S., Carriere, J. F., and Collins, M. T. (1988). A model for analyzing growth kinetics of a slowly growing Mycobacterium sp. Appl. Environ. Microbiol. 54, 910-916.

Lambrecht, R. S., and Collins, M. T. (1992). Mycobacterium paratuberculosis. Factors that influence mycobactin dependence. Diagn. Microbiol. Infect. Dis. 15, 239-246. doi: 10.1016/0732-8893(92)90119-E

Lamont, E. A., and Sreevatsan, S. (2010). Paradigm redux-Mycobacterium avium subspecies paratuberculosis-macrophage interactions show clear variations between bovine and human physiological body temperatures. Microb. Pathog. 48, 143-149. doi: 10.1016/j.micpath.2010.02.002

Lamont, E. A., Talaat, A. M., Coussens, P. M., Bannantine, J. P., Grohn, Y. T., Katani, R., et al. (2014). Screening of Mycobacterium avium subsp. paratuberculosis mutants for attenuation in a bovine monocyte derived macrophage model. Front. Cell. Infect. Microbiol. 4:87. doi: 10.3389/fcimb.2014.00087

Li, L., Bannantine, J. P., Zhang, Q., Amonsin, A., May, B. J., Alt, D., et al. (2005). The complete genome sequence of Mycobacterium avium subspecies paratuberculosis. Proc. Natl. Acad. Sci. U.S.A. 102, 12344-12349. doi: 10.1073/pnas.0505662102

Li, L., Munir, S., Bannantine, J. P., Sreevatsan, S., Kanjilal, S., and Kapur, V. (2007). Rapid expression of Mycobacterium avium subsp. paratuberculosis recombinant proteins for antigen discovery. Clin. Vaccine Immunol. 14, 102-105. doi: 10.1128/CVI.00138-06

Livneh, A., Golan, L., Rosenshine, I., Zinniel, D. K., Chahal, H. K., Chacon, O., et al. (2005). "In vivo and in vitro characterization of Mycobacterium avium subsp. paratuberculosis (MAP) mutants," in Proceeding of the 8th International Colloquium on Paratuberculosis (Copenhagen: International Association for Paratuberculosis Inc.).

Lu, Z., Schukken, Y. H., Smith, R. L., and Grohn, Y. T. (2013). Using vaccination to prevent the invasion of Mycobacterium avium subsp. paratuberculosis in dairy herds: a stochastic simulation study. Prev. Vet. Med. 110, 335-345. doi: 10.1016/j.prevetmed.2013.01.006

McAdam, R. A., Quan, S., Smith, D. A., Bardarov, S., Betts, J. C., Cook, F. C., et al. (2002). Characterization of a Mycobacterium tuberculosis H37Rv transposon library reveals insertions in 351 ORFs and mutants with altered virulence. Microbiology 148, 2975-2986.

McAdam, R. A., Weisbrod, T. R., Martin, J., Scuderi, J. D., Brown, A. M., Cirillo, J. D., et al. (1995). In vivo growth characteristics of leucine and methionine auxotrophic mutants of Mycobacterium bovis BCG generated by transposon mutagenesis. Infect. Immun. 63, 1004-1012.

Murcia, M. I., Garcia, M. J., Otal, I., Gomez, A. B., and Menendez, M. C. (2007). Molecular features of Mycobacterium avium human isolates carrying a single copy of IS1245 and IS1311 per genome. FEMS Microbiol. Lett. 272, 229-237. doi: 10.1111/j.1574-6968.2007.00769.x

Nagata, R., Muneta, Y., Yoshihara, K., Yokomizo, Y., and Mori, Y. (2005). Expression cloning of gamma interferon-inducing antigens of Mycobacterium avium subsp. paratuberculosis. Infect. Immun. 73, 3778-3782. doi: 10.1128/IAI.73.6.37783782.2005

Okkels, L. M., Brock, I., Follmann, F., Agger, E. M., Arend, S. M., Ottenhoff, T. H., et al. (2003). PPE protein (Rv3873) from DNA segment RD1 of Mycobacterium tuberculosis: strong recognition of both specific T-cell epitopes and epitopes conserved within the PPE family. Infect. Immun. 71, 6116-6123. doi: 10.1128/IAI.71.11.6116-6123.2003

Ott, S. L., Wells, S. J., and Wagner, B. A. (1999). Herd-level economic losses associated with Johne's Disease on US dairy operations. Prev. Vet. Med. 40, 179-192. doi: 10.1016/S0167-5877(99)00037-9

Park, K. T., Dahl, J. L., Bannantine, J. P., Barletta, R. G., Ahn, J., Allen, A. J., et al. (2008). Demonstration of allelic exchange in the slow-growing bacterium Mycobacterium avium subsp. paratuberculosis, and generation of mutants with deletions at the pknG, relA, and lsr2 loci. Appl. Environ. Microbiol. 74, 1687-1695. doi: 10.1128/AEM.01208-07

Reddy, V. M., Luna-Herrera, J., and Gangadharam, P. R. (1996). Pathobiological significance of colony morphology in Mycobacterium avium complex. Microb. Pathog. 21, 97-109. doi: 10.1006/mpat.1996.0046

Riley, R., Pellegrini, M., and Eisenberg, D. (2008). Identifying cognate binding pairs among a large set of paralogs: the case of PE/PPE proteins of Mycobacterium tuberculosis. PLoS Comput. Biol. 4:e1000174. doi: 10.1371/journal.pcbi. 1000174

Sampson, S. L., Dascher, C. C., Sambandamurthy, V. K., Russell, R. G., Jacobs, W. R. Jr., Bloom, B. R., et al. (2004). Protection elicited by a double leucine and 
pantothenate auxotroph of Mycobacterium tuberculosis in guinea pigs. Infect. Immun. 72, 3031-3037. doi: 10.1128/IAI.72.5.3031-3037.2004

Sassetti, C. M., and Rubin, E. J. (2003). Genetic requirements for mycobacterial survival during infection. Proc. Natl. Acad. Sci. U.S.A. 100, 12989-12994. doi: 10.1073/pnas.2134250100

Scandurra, G. M., De Lisle, G. W., Cavaignac, S. M., Young, M., Kawakami, R. P., and Collins, D. M. (2010). Assessment of live candidate vaccines for paratuberculosis in animal models and macrophages. Infect. Immun. 78, 1383-1389. doi: 10.1128/IAI.01020-09

Scandurra, G. M., Young, M., De Lisle, G. W., and Collins, D. M. (2009). A bovine macrophage screening system for identifying attenuated transposon mutants of Mycobacterium avium subsp. paratuberculosis with vaccine potential. J. Microbiol. Methods 77, 58-62. doi: 10.1016/j.mimet.2009.01.005

Schwyn, B., and Neilands, J. B. (1987). Universal chemical assay for the detection and determination of siderophores. Anal. Biochem. 160, 47-56. doi: 10.1016/0003-2697(87)90612-9

Shin, S. J., Wu, C. W., Steinberg, H., and Talaat, A. M. (2006). Identification of novel virulence determinants in Mycobacterium paratuberculosis by screening a library of insertional mutants. Infect. Immun. 74, 3825-3833. doi: 10.1128/IAI. 01742-05

Stabel, J. R. (1998). Johne's Disease: a hidden threat. J. Dairy Sci. 81, 283-288. doi: 10.3168/jds.S0022-0302(98)75577-8

Stabel, J. R., and Stabel, T. J. (1995). Immortalization and characterization of bovine peritoneal macrophages transfected with SV40 plasmid DNA. Vet. Immunol. Immunopathol. 45, 211-220. doi: 10.1016/0165-2427(94)05348-V

Stewart, G. R., Wernisch, L., Stabler, R., Mangan, J. A., Hinds, J., Laing, K. G., et al. (2002). Dissection of the heat-shock response in Mycobacterium tuberculosis using mutants and microarrays. Microbiology 148, 3129-3138.

Sweeney, R. W. (1996). Transmission of paratuberculosis. Vet. Clin. North Am. Food Anim. Pract. 12, 305-312.

Wang, J., Pritchard, J. R., Kreitmann, L., Montpetit, A., and Behr, M. A. (2014). Disruption of Mycobacterium avium subsp. paratuberculosis-specific genes impairs in vivo fitness. BMC Genomics 15:415. doi: 10.1186/1471-2164-15-415

Woo, S. R., Sotos, J., Hart, A. P., Barletta, R. G., and Czuprynski, C. J. (2006). Bovine monocytes and a macrophage cell line differ in their ability to phagocytose and support the intracellular survival of Mycobacterium avium subsp. paratuberculosis. Vet. Immunol. Immunopathol. 110, 109-120. doi: 10.1016/j.vetimm.2005.09.010

Wu, C. W., Glasner, J., Collins, M., Naser, S., and Talaat, A. M. (2006). Whole-genome plasticity among Mycobacterium avium subspecies: insights from comparative genomic hybridizations. J. Bacteriol. 188, 711-723. doi: 10.1128/JB.188.2.711-723.2006

Wu, C. W., Schramm, T. M., Zhou, S., Schwartz, D. C., and Talaat, A. M. (2009). Optical mapping of the Mycobacterium avium subspecies paratuberculosis genome. BMC Genomics 10:25. doi: 10.1186/1471-2164-10-25

Zhao, B., Collins, M. T., and Czuprynski, C. J. (1997). Effects of gamma interferon and nitric oxide on the interaction of Mycobacterium avium subsp. paratuberculosis with bovine monocytes. Infect. Immun. 65, 1761-1766.

Zhao, B. Y., Czuprynski, C. J., and Collins, M. T. (1999). Intracellular fate of Mycobacterium avium subspecies paratuberculosis in monocytes from normal and infected, interferon-responsive cows as determined by a radiometric method. Can. J. Vet. Res. 63, 56-61.

Zheng, Y. T., Toyofuku, M., Nomura, N., and Shigeto, S. (2013). Correlation of carotenoid accumulation with aggregation and biofilm development in Rhodococcus sp. SD-74. Anal. Chem. 85, 7295-7301. doi: 10.1021/ac401188f

Conflict of Interest Statement: Patent: Methods for the identification of Virulence Determinants. Raúl G. Barletta, N. Beth Harris. U.S. Patent No. 7,740,867, Granted June 22, 2010. The Review Editor, Jeffrey Cirillo, declares that, despite having collaborated with authors Raul Barletta and Denise Kinniel, the review process was handled objectively. The authors declare that the research was conducted in the absence of any commercial or financial relationships that could be construed as a potential conflict of interest.

Received: 30 May 2014; accepted: 25 September 2014; published online: 15 October 2014.

Citation: Rathnaiah G, Lamont EA, Harris NB, Fenton RJ, Zinniel DK, Liu X, Sotos J, Feng Z, Livneh-Kol A, Shpigel NY, Czuprynski CJ, Sreevatsan S and Barletta RG (2014) Generation and screening of a comprehensive Mycobacterium avium subsp. paratuberculosis transposon mutant bank. Front. Cell. Infect. Microbiol. 4:144. doi 10.3389/fcimb.2014.00144

This article was submitted to the journal Frontiers in Cellular and Infection Microbiology.

Copyright (c) 2014 Rathnaiah, Lamont, Harris, Fenton, Zinniel, Liu, Sotos, Feng, Livneh-Kol, Shpigel, Czuprynski, Sreevatsan and Barletta. This is an open-access article distributed under the terms of the Creative Commons Attribution License (CC BY). The use, distribution or reproduction in other forums is permitted, provided the original author(s) or licensor are credited and that the original publication in this journal is cited, in accordance with accepted academic practice. No use, distribution or reproduction is permitted which does not comply with these terms. 